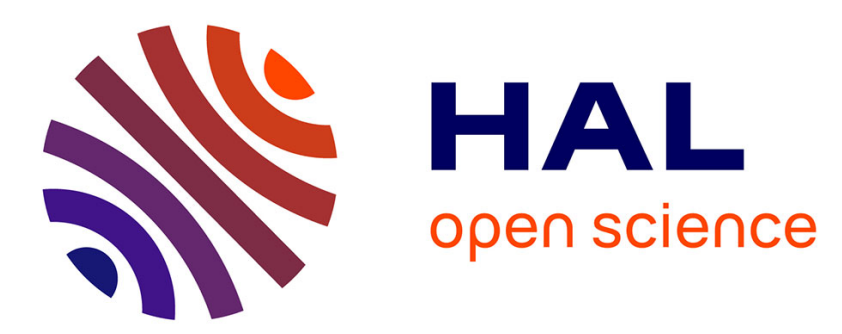

\title{
Vibro-impact of a plate on rigid obstacles: existence theorem, convergence of a scheme and numerical simulations
}

Cédric Pozzolini, Yves Renard, Michel Salaün

\section{To cite this version:}

Cédric Pozzolini, Yves Renard, Michel Salaün. Vibro-impact of a plate on rigid obstacles: existence theorem, convergence of a scheme and numerical simulations. IMA Journal of Numerical Analysis, 2013, vol. 33, pp. 261 -294. 10.1093/imanum/drr057 . hal-00812715

HAL Id: hal-00812715

https://hal.science/hal-00812715

Submitted on 12 Apr 2013

HAL is a multi-disciplinary open access archive for the deposit and dissemination of scientific research documents, whether they are published or not. The documents may come from teaching and research institutions in France or abroad, or from public or private research centers.
L'archive ouverte pluridisciplinaire HAL, est destinée au dépôt et à la diffusion de documents scientifiques de niveau recherche, publiés ou non, émanant des établissements d'enseignement et de recherche français ou étrangers, des laboratoires publics ou privés. 


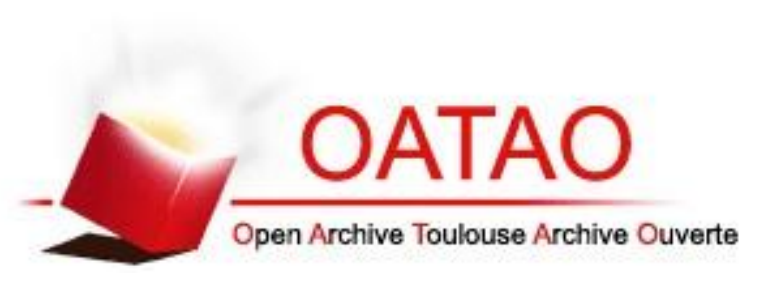

\section{Open Archive Toulouse Archive Ouverte (OATAO)}

OATAO is an open access repository that collects the work of Toulouse researchers and makes it freely available over the web where possible.

This is an author-deposited version published in: http://oatao.univ-toulouse.fr/ Eprints ID: 8874

To link to this article: DOI: $10.1093 / \mathrm{imanum} / \mathrm{drr} 057$

URL: http://dx.doi.org/10.1093/imanum/drr057

To cite this version: Pozzolini, Cédric and Renard, Yves and Salaün, Michel Vibro-impact of a plate on rigid obstacles: existence theorem, convergence of a scheme and numerical simulations. (2013) IMA Journal of Numerical Analysis, vol. 33 ( $\mathrm{n}^{\circ}$ 1). pp. 261 -294. ISSN 0272-4979 


\title{
Vibro-impact of a plate on rigid obstacles: existence theorem, convergence of a scheme and numerical simulations
}

\author{
C. Pozzolini \\ Université de Lyon, CNRS, INSA-Lyon, ICJ UMR5208, F-69621 Villeurbanne, France \\ cedric.pozzolini@insa-lyon.fr \\ Y. RENARD \\ Université de Lyon, CNRS, INSA-Lyon, ICJ UMR5208, LaMCoS UMR5259, \\ F-69621 Villeurbanne, France \\ yves.renard@insa-lyon.fr \\ AND \\ M. SALAUN* \\ Université de Toulouse, INSA, UPS, EMAC, ISAE; ICA (Institut Clément Ader), \\ 10 Avenue Edouard Belin, F-31055 Toulouse, France \\ *Corresponding author: michel.salaun@isae.fr
}

\begin{abstract}
The purpose of this paper is to describe a fully discrete approximation and its convergence to the continuum dynamical impact problem for the fourth-order Kirchhoff-Love plate model with nonpenetration Signorini contact condition. We extend to the case of plates the theoretical results of weak convergence due to Y. Dumont and L. Paoli, which was stated for Euler-Bernouilli beams. In particular, this provides an existence result for the solution of this problem. Finally, we discuss the numerical results we obtain.
\end{abstract}

Keywords: variational inequalities; finite element method; elastic plate; dynamics with unilateral constraints; scheme convergence.

\section{Introduction}

The impact of linear elastic thin structures, such as beams, membranes or plates, is a domain where there are still fundamental open questions despite a rather important literature. This includes, in particular, the existence and uniqueness of solutions, the convergence and stability of numerical schemes, the modelling of a restitution coefficient and the construction of energy-conserving schemes.

In the particular case of the vibro-impact problem between an Euler-Bernouilli beam and a rigid obstacle, an existence result was shown by Dumont \& Paoli (2006). They established the convergence of the solution of a fully discrete problem to the continuum model. But there were no results on whether energy is conserved in the limit or not. Indeed, it can be easily shown that uniqueness does not hold for this system (see Ahn \& Stewart, 2005, for a counterexample). Moreover, it is generally not possible to prove that each solution to this problem is energy conserving. This is due to the weak regularity involved since, in particular, velocities may be discontinuous.

The dynamic contact problem for von Karman plates is studied in Bock \& Jarušek (2008a,b). In the first paper the authors show the existence of a solution, using penalization techniques, while other 
existence results are given in the second paper by the introduction of a viscosity term. Here our main goal is to extend the Dumont and Paoli results to the case of Kirchhoff-Love plates. We present a convergence result for a fully discrete scheme towards one solution of the continuous problem. This establishes both an existence result for the solution of the continuous problem and ensures that one subsequence weakly converges towards this solution. We do not establish a uniqueness result. Such a result would certainly require the ability to express an additional impact law (see Paoli \& Schatzman, 2007; Paoli, 2001). Although the consideration of an impact law is something very natural for the modelling of rigid-body impacts, this concept seems to be rather difficult to extend to the framework of thin deformable bodies, especially with regard to the discretization.

The paper is organized as follows. In the next section an elastodynamical Kirchhoff-Love plate model is described, as well as the vibro-impact model. In Section 3 the fully discrete approximation of the problem (finite element model and time scheme) is introduced. Section 4 gives the most important result of this paper, namely a convergence result for fully discrete schemes. Finally, in Section 5 we present and discuss some numerical experiments.

\section{Notation and statement of the problem}

\subsection{Variational formulation of the plate model}

Let us consider a thin elastic plate, i.e., a plane structure for which one dimension, called the thickness, is very small compared to the others. For this kind of structure, starting from a priori hypotheses on the expression of the displacement fields, a two-dimensional problem is usually derived from the three-dimensional elasticity formulation by means of integration along the thickness. Then the unknown variables are set down on the midplane of the plate.

Let $\Omega$ be an open, bounded, connected subset of the plane $\mathbb{R}^{2}$, with Lipschitz boundary. It will define the middle plane of the plate. Then the plate in its stress-free reference configuration coincides with domain

$$
\left.\Omega^{\varepsilon}=\Omega \times\right]-\varepsilon,+\varepsilon\left[=\left\{\left(x_{1}, x_{2}, x_{3}\right) \in \mathbb{R}^{3} /\left(x_{1}, x_{2}\right) \in \Omega \text { and } x_{3} \in\right]-\varepsilon ; \varepsilon[\},\right.
$$

where $2 \varepsilon>0$ is called the thickness.

In plate theory, it is usual to consider the following approximation of the three-dimensional displacements for $\left(x_{1}, x_{2}, x_{3}\right) \in \Omega^{\varepsilon}$ :

$$
\left\{\begin{array}{l}
u_{1}\left(x_{1}, x_{2}, x_{3}\right)=\bar{u}_{1}\left(x_{1}, x_{2}\right)+x_{3} \psi_{1}\left(x_{1}, x_{2}\right), \\
u_{2}\left(x_{1}, x_{2}, x_{3}\right)=\bar{u}_{2}\left(x_{1}, x_{2}\right)+x_{3} \psi_{2}\left(x_{1}, x_{2}\right), \\
u_{3}\left(x_{1}, x_{2}, x_{3}\right)=u_{3}\left(x_{1}, x_{2}\right) .
\end{array}\right.
$$

In these expressions, $\bar{u}_{1}$ and $\bar{u}_{2}$ are the membrane displacements of the midplane points, $u_{3}$ is the deflection, while $\psi_{1}$ and $\psi_{2}$ are the section rotations. In the case of a homogeneous isotropic material, the variational plate model splits into two independent problems: the first, called the membrane problem, deals only with membrane displacements, while the second, called the bending problem, concerns deflection and rotations. In this paper we shall only address the bending problem, and we shall consider the Kirchhoff-Love model, which can be seen as a particular case of (2.1) obtained by introducing the so-called Kirchhoff-Love assumptions:

$$
\psi=-\nabla u_{3} \Leftrightarrow\left\{\begin{array}{l}
\psi_{1}=-\partial_{1} u_{3}, \\
\psi_{2}=-\partial_{2} u_{3}
\end{array}\right.
$$


where $\partial_{\alpha}$ stands for the partial derivative with respect to $x_{\alpha}$, for $\alpha=1$ or 2 . Consequently, the deflection is the only unknown for the bending Kirchhoff-Love plate problem. For convenience, it will be denoted by $u$ for the remainder of this paper. As far as loading is concerned, the plate is subject to a volume force $F$ and two surface forces, say $G^{+}$and $G^{-}$, applied on the top and bottom surfaces. Then, if we assume that the previous forces are purely perpendicular to the midplane, the resulting transverse loading is

$$
f_{R}=G_{3}^{+}+G_{3}^{-}+\int_{-\varepsilon}^{\varepsilon} F_{3} \mathrm{~d} x_{3}
$$

where $G_{3}^{+}, G_{3}^{-}$and $F_{3}$ are, respectively, the third components of $G^{+}, G^{-}$and $F$. So, the variational formulation of the Kirchhoff-Love elastodynamical model for a thin elastic clamped/free plate is:

$$
\begin{aligned}
& \text { find } u=u(x, t) \text { with }(x, t) \in \Omega \times[0, T] \text { such that for any } w \in \mathbb{V} \\
& \qquad \int_{\Omega} \partial_{t t}^{2} u(x, t) w(x) \mathrm{d} x+\int_{\Omega} \frac{D}{2 \rho \varepsilon}\left[(1-v) \partial_{\alpha \beta}^{2} u+v \Delta u \delta_{\alpha \beta}\right] \partial_{\alpha \beta}^{2} w \mathrm{~d} x=\int_{\Omega} f w \mathrm{~d} x
\end{aligned}
$$

with $f=\frac{f_{R}}{2 \rho \varepsilon}$ ( $\rho$ and $\varepsilon$ are assumed to be constant all along the plate), and

$$
u(x, 0)=u_{0}(x), \quad \partial_{t} u(x, 0)=v_{0}(x), \quad \forall x \in \Omega,
$$

where $\partial_{t t}^{2} u=\frac{\partial^{2} u}{\partial t^{2}}, \partial_{\alpha \beta}^{2} u=\frac{\partial^{2} u}{\partial x_{\alpha} \partial x_{\beta}}$, and the bending modulus is $D=\frac{2 E \varepsilon^{3}}{3\left(1-v^{2}\right)}$ for a plate made of a homogeneous and isotropic material, for which the mechanical constants are its Young's modulus $E$, Poisson's ratio $v$ and mass density $\rho$. As usual, we have $E>0,0<v<0.5$ and $\rho>0$. Moreover, $\delta_{\alpha \beta}$ is the Kronecker symbol and the summation convention over repeated indices is adopted, Greek indices varying in $\{1,2\}$. The plate is assumed to be clamped on a nonzero Lebesgue-measure part of the boundary $\partial \Omega$ denoted $\Gamma_{\mathrm{c}}$ and free on $\Gamma_{\mathrm{f}}$, such that $\partial \Omega=\Gamma_{\mathrm{c}} \cup \Gamma_{\mathrm{f}}$. Then the space of admissible displacements is

$$
\mathbb{V}=\left\{w \in H^{2}(\Omega) / w(x)=0=\partial_{\mathrm{n}} w(x) \quad \forall x \in \Gamma_{\mathrm{c}}\right\},
$$

where $\partial_{\mathrm{n}} w$ is the normal derivative along $\Gamma_{\mathrm{c}}$.

In order to guarantee that (2.2) is well posed, we use the following result.

Lemma 2.1 The bilinear form $a: \mathbb{V} \times \mathbb{V} \rightarrow \mathbb{R}$ defined by

$$
a(u, v)=\int_{\Omega} \frac{D}{2 \rho \varepsilon}\left[(1-v) \partial_{\alpha \beta}^{2} u+v \Delta u \delta_{\alpha \beta}\right] \partial_{\alpha \beta}^{2} v \mathrm{~d} x
$$

is a scalar product on $\mathbb{V}$, which is equivalent to the canonical scalar product of $H^{2}(\Omega)$ defined on $\mathbb{V}$.

The bilinear map $a$ is obviously continuous in $\mathbb{V}$. Then there exists a strictly positive constant, say $M$, such that for any $u \in \mathbb{V}$ then $a(u, u) \leqslant M\|u\|_{\mathbb{V}}^{2}$. The converse inequality is due to the coercivity of $a(\cdot, \cdot)$, which can be established using Petree-Tartar's lemma which we recall here.

Lemma 2.2 (Ern \& Guermond, 2004) Let X, Y, $\mathrm{Z}$ be three Banach spaces, $A \in \mathcal{L}(\mathrm{X}, \mathrm{Y})$ injective, $T \in \mathcal{L}(\mathrm{X}, \mathrm{z})$ compact. If there exists $c>0$ such that $c\|x\|_{\mathrm{X}} \leqslant\|A x\|_{\mathrm{Y}}+\|T x\|_{\mathrm{Z}}$ for any $x \in \mathrm{X}$, then there exists $\alpha>0$ such that

$$
\alpha\|x\|_{\mathrm{X}} \leqslant\|A x\|_{\mathrm{Y}} \quad \forall x \in \mathrm{X} .
$$


Proof (Lemma 2.1). Let us remark that

$$
\begin{aligned}
a(u, u) & =\int_{\Omega} \frac{D}{\Omega \rho \varepsilon}\left[(1-v) \partial_{\alpha \beta}^{2} u+v \Delta u \delta_{\alpha \beta}\right] \partial_{\alpha \beta}^{2} u \mathrm{~d} x \\
& =\int_{\Omega} \frac{D}{2 \rho \varepsilon}\left[(1-v) \partial_{\alpha \beta}^{2} u \partial_{\alpha \beta}^{2} u+v(\Delta u)^{2}\right] \mathrm{d} x \\
& \geqslant \frac{(1-v) D}{2 \rho \varepsilon} \int_{\Omega} \partial_{\alpha \beta}^{2} u \partial_{\alpha \beta}^{2} u \mathrm{~d} x \text { as } v>0 \\
= & \frac{(1-v) D}{2 \rho \varepsilon}\|\operatorname{Hess}(u)\|_{\mathrm{Y}}^{2},
\end{aligned}
$$

Hess $(u)$ being the Hessian matrix of $u$ and with $\mathrm{Y}=\left(L^{2}(\Omega)\right)^{4}$. Now Petree-Tartar's lemma is applied with $A$ defined from $\mathrm{X}=\mathbb{V}$ to $\mathrm{Y}$ by $A u=\operatorname{Hess}(u)$, which is injective because of the boundary conditions, $\Gamma_{\mathrm{c}}$ having a nonzero measure in $\partial \Omega$. Setting $\mathrm{z}=H^{1}(\Omega)$ and $T=i d_{\mathrm{x}, \mathrm{z}}$, which is compact, we obtain the $\mathbb{V}$-coercivity of $A$, and consequently of $a$, as $v<1$.

\subsection{Vibro-impact formulation of the plate model}

Let us now introduce the dynamic frictionless Kirchhoff-Love equation with Signorini contact conditions along the plate. We assume that the plate motion is limited by rigid obstacles, located above and below the plate (see Fig. 1). So, the displacement is constrained to belong to the convex set $\mathbb{K} \subset \mathbb{V}$ given by

$$
\mathbb{K}=\left\{v \in \mathbb{V} / g_{1}(x) \leqslant v(x) \leqslant g_{2}(x) \quad \forall x \in \Omega\right\},
$$

where $g_{1}$ and $g_{2}$ are two mappings from $\Omega$ to $\overline{\mathbb{R}}:=\mathbb{R} \cup\{-\infty,+\infty\}$ such that there exists $g>0$ with

$$
g_{1}(x) \leqslant-g<0<g \leqslant g_{2}(x) \quad \forall x \in \Omega .
$$

Since impact will occur in this system, classical regular solutions cannot be expected. In particular, the velocities may be discontinuous. To set the weak formulation, the following function spaces are

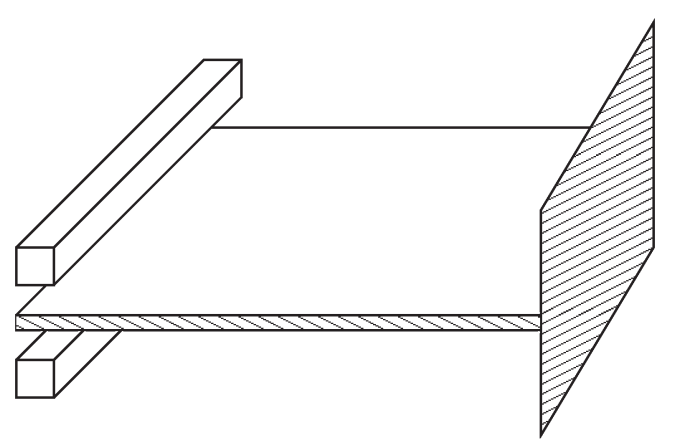

FIG. 1. Example of bending a clamped plate between rigid obstacles. 
introduced:

$$
\begin{aligned}
\mathbb{H} & =L^{2}(\Omega), \\
\mathbb{V} & =\left\{w \in H^{2}(\Omega) / w(x)=0=\partial_{\mathrm{n}} w(x) \quad \forall x \in \Gamma_{\mathrm{c}}\right\}, \\
\tilde{\mathbb{U}} & =\left\{w \in L^{2}(0, T ; \mathbb{K}), \dot{w} \in L^{2}\left(0, T ; L^{2}(\Omega)\right)\right\}, \\
\mathbb{U} & =\left\{w \in L^{\infty}(0, T ; \mathbb{K}), \dot{w} \in L^{\infty}\left(0, T ; L^{2}(\Omega)\right)\right\},
\end{aligned}
$$

where $\dot{w}=\partial_{t} w=\frac{\partial w}{\partial t}$, and $T>0$ is the length of the plate-motion study. The norm in $\mathbb{H}$ will be denoted by $|\cdot| \mathbb{H}$.

The frictionless elastodynamic problem for a plate between two rigid obstacles consists in finding $u \in \tilde{\mathbb{U}}$ with $u(\cdot, 0)=u_{0}$ in $\mathbb{K}$ and $\dot{u}(\cdot, 0)=v_{0}$ such that

$$
\begin{aligned}
& -\int_{0}^{T} \int_{\Omega} \partial_{t} u \partial_{t}(\tilde{w}-u) \mathrm{d} x \mathrm{~d} t+\int_{0}^{T} a(u(\cdot, t),(\tilde{w}-u)(\cdot, t)) \mathrm{d} t \\
& \quad \geqslant \int_{\Omega} v_{0}(x)\left(\tilde{w}(x, 0)-u_{0}(x)\right) \mathrm{d} x+\int_{0}^{T} \int_{\Omega} f(\tilde{w}-u) \mathrm{d} x \mathrm{~d} t \quad \forall \tilde{w} \in \tilde{\mathbb{U}}, \tilde{w}(\cdot, T)=u(\cdot, T) .
\end{aligned}
$$

REMARK 2.3 The discretization of (2.8) does not describe the motion completely (Paoli, 2001); in addition, it would require an impact law. For an impact at $\left(x_{0}, t_{0}\right)$, this law is given by a relation between velocities before and after impact, as

$$
\frac{\partial u}{\partial t}\left(x_{0}, t_{0}^{+}\right)=-e \frac{\partial u}{\partial t}\left(x_{0}, t_{0}^{-}\right),
$$

where $e$ belongs to $[0,1]$. Since one can only guarantee that the velocity is $L^{2}(\Omega)$ in space, it is not easy to express (2.9) rigorously. Moreover, in Paoli \& Schatzman (2007), the authors observe that the restitution coefficient for a bar is a rather ill-defined concept. They observed that the apparent restitution coefficient depends very strongly on the initial angle of the bar with the horizontal. In the particular case of a slender bar dropped on a rigid foundation, the chosen value of the restitution coefficient does not seem to have great influence on the limit displacement when the space step tends to zero, as has been shown in Paoli \& Schatzman (2007). The idea to explicitly incorporate the restitution coefficient into (2.8) seems a rather problematic task since the postimpact normal velocity at a point due to the impact force would need to be separated from the postimpact normal velocity due to elastic waves. Therefore, knowing whether our schemes will simulate the experimental behaviour is an interesting question.

\section{Full discretization of the problem}

\subsection{Finite element model for the plate problem}

Let us begin with the space discretization of the displacement. The Kirchhoff-Love model corresponds to a fourth-order partial differential equation. Consequently, a conformal finite element method needs the use of $\mathcal{C}^{1}$ (continuously differentiable) finite elements. Here we consider the classical Argyris triangle, which uses $P_{5}$ polynomials, and the Fraeijs de Veubeke-Sanders quadrilateral (reduced FVS); 


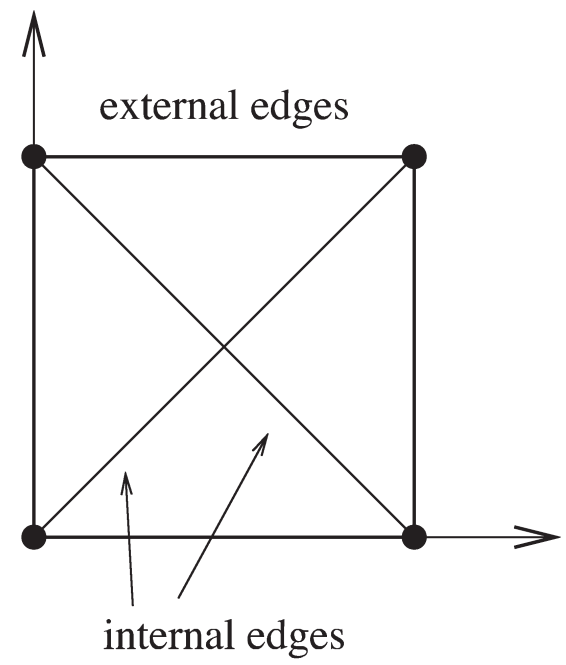

FIG. 2. FVS quadrilateral. Location of degrees of freedom and subtriangles.

see Ciarlet (1978). For the FVS element, the quadrilateral is divided into four subtriangles (see Fig. 2). The basis functions are $P_{3}$ polynomials on each subtriangle and matched $\mathcal{C}^{1}$ across each internal edge. In addition, to decrease the number of degrees of freedom, the normal derivative is assumed to vary linearly along the external edges of the element (this assumption does not hold on the internal edges). Finally, for FVS quadrilaterals, there are only three degrees of freedom at each node: the value of the function and its first derivatives. Let us assume from now on that $h>0$ stands for the mesh parameter and that $\mathbb{V}^{h}$ is a finite-dimensional subspace of $\mathbb{V}$ built using the previous finite element methods. Then, following Ciarlet (1978) for Argyris triangles and Ciarlet (1991) for FVS quadrilaterals, for all $w \in \mathbb{V}$ there exists a sequence $\left(w^{h}\right)_{h>0}$ of elements of $\mathbb{V}^{h}$ such that

$$
\left\|w^{h}-w\right\|_{\mathbb{V}} \rightarrow 0 \quad \text { when } h \rightarrow 0
$$

Finally, let us remark that there also exist some nonconformal approximations (see Brenner et al., 2012), but we do not use them here because we develop our theory within the frame of conformal methods.

\subsection{Time discretization}

Now we consider the time discretization of problem (2.8). For $N \in \mathbb{N}^{*}$, the time step is denoted by $\Delta t=T / N$. The time scheme is initialized by choosing $u_{0}^{h}$ and $u_{1}^{h}$ in $\mathbb{K}^{h}=\mathbb{K} \cap \mathbb{V}^{h}$ such that

$$
\lim _{h \rightarrow 0, \Delta t \rightarrow 0}\left\|u_{0}^{h}-u_{0}\right\|_{\mathbb{V}}+\left|\frac{u_{1}^{h}-u_{0}^{h}}{\Delta t}-v_{0}\right|_{\mathbb{H}}=0 .
$$

As far as the loading is concerned, we assume that $f$ belongs to $L^{2}\left(0, T ; L^{2}(\Omega)\right)$. Then, for all $x \in \Omega$ and $n \in\{1, \ldots, N-1\}$, we set

$$
f_{n}(x)=\frac{1}{\Delta t} \int_{n \Delta t}^{(n+1) \Delta t} f(x, s) \mathrm{d} s .
$$


For time discretization, we consider the corresponding fully discrete scheme, which consists in finding $u_{n+1}^{h}$ for all $n \in\{2, \ldots, N-1\}$, the solution of:

$$
\left\{\begin{array}{l}
\text { find } u_{n+1}^{h} \in \mathbb{K}^{h} \text { such that } \\
\left(w-u_{n+1}^{h}\right)^{\mathrm{T}} \mathbf{M}\left(\frac{u_{n+1}^{h}-2 u_{n}^{h}+u_{n-1}^{h}}{(\Delta t)^{2}}\right)+\left(w-u_{n+1}^{h}\right)^{\mathrm{T}} \mathbf{K}\left(\beta u_{n+1}^{h}+(1-2 \beta) u_{n}^{h}+\beta u_{n-1}^{h}\right) \\
\geqslant\left(w-u_{n+1}^{h}\right)^{\mathrm{T}} f^{n \beta},
\end{array}\right.
$$

which is a classical Newmark scheme with parameters $\beta$ and $\gamma=1 / 2$. If $\left(\psi_{i}\right)_{i}$ denotes the finite element basis functions, then in the previous expression:

- $f^{n \beta}$ is the loading, where

$$
f_{i}^{n \beta}=\int_{\Omega}\left(\beta f_{n+1}+(1-2 \beta) f_{n}+\beta f_{n-1}\right) \psi_{i} \mathrm{~d} x ;
$$

- $\mathbf{M}$ is the mass matrix, where

$$
\mathbf{M}_{i j}=\int_{\Omega} \psi_{i} \psi_{j} \mathrm{~d} x
$$

- $\mathbf{K}$ is the rigidity matrix, where

$$
\mathbf{K}_{i j}=a\left(\psi_{i}, \psi_{j}\right) .
$$

Let us remark that the previous inequality is also equivalent to the inclusion:

$$
\left\{\begin{array}{l}
\text { find } u_{n+1}^{h} \in \mathbb{K}^{h} \text { such that } \\
\left(\mathbf{M}+\beta\left(\Delta t^{2}\right) \mathbf{K}\right) u_{n+1}^{h}+\left(\Delta t^{2}\right) \partial \mathbb{I}_{\mathbb{K}^{h}}\left(u_{n+1}^{h}\right) \ni f_{n}^{h} \\
\text { where } \\
f_{n}^{h}=\left(2 \mathbf{M}-(1-2 \beta)\left(\Delta t^{2} \mathbf{K}\right) u_{n}^{h}-\left(\mathbf{M}+\beta\left(\Delta t^{2} \mathbf{K}\right) u_{n-1}^{h}+(\Delta) t^{2} f^{n \beta}\right.\right.
\end{array}\right.
$$

As $\mathbb{K}^{h}$ is a nonempty, closed, convex subset of $\mathbb{W}^{h}$ and thanks to Lemma 2.1 , we easily obtain by induction on $n$ that $u_{n+1}^{h}$ is uniquely defined for all $n \in\{1, \ldots, N-1\}$. This kind of variational inequality has been intensively studied by Paoli and Schatzman (see Paoli, 2001; Paoli \& Schatzman, 2007).

\section{A convergence result for a Newmark-Dumont-Paoli-type scheme}

The discrete problem associated to (3.3) is:

$$
\left\{\begin{array}{l}
\text { find } u_{n+1}^{h} \in \mathbb{K}^{h} \text { such that for all } w^{h} \in \mathbb{K}^{h} \\
\int_{\Omega} \frac{u_{n+1}^{h}-2 u_{n}^{h}+u_{n-1}^{h}}{(\Delta t)^{2}}\left(w^{h}-u_{n+1}^{h}\right) \mathrm{d} x+a\left(\beta u_{n+1}^{h}+(1-2 \beta) u_{n}^{h}+\beta u_{n-1}^{h}, w^{h}-u_{n+1}^{h}\right) \\
\geqslant \int_{\Omega}\left[\beta f_{n+1}+(1-2 \beta) f_{n}+\beta f_{n-1}\right]\left(w^{h}-u_{n+1}^{h}\right) \mathrm{d} x .
\end{array}\right.
$$


Dumont \& Paoli (2006) studied the same kind of problem, corresponding to a fully discrete beam problem. They established unconditional stability and gave a convergence result for $\beta=1 / 2$, whereas a conditional stability result is obtained when $\beta \in[0,1 / 2$ [. In the following, we shall adapt their proof to the case of a Kirchhoff-Love plate, restricting ourselves to the case $\beta=1 / 2$. So the fully discrete scheme we consider is:

$$
\left\{\begin{array}{l}
\text { find } u_{n+1}^{h} \in \mathbb{K}^{h} \text { such that for all } w^{h} \in \mathbb{K}^{h} \\
\int_{\Omega} \frac{u_{n+1}^{h}-2 u_{n}^{h}+u_{n-1}^{h}}{(\Delta t)^{2}}\left(w^{h}-u_{n+1}^{h}\right) \mathrm{d} x+a\left(\frac{u_{n+1}^{h}+u_{n-1}^{h}}{2}, w^{h}-u_{n+1}^{h}\right) \\
\geqslant \int_{\Omega} \frac{f_{n+1}+f_{n-1}}{2}\left(w^{h}-u_{n+1}^{h}\right) \mathrm{d} x
\end{array}\right.
$$

The following result, which states that the discrete solution is uniformly bounded in time, is straightforwardly obtained by adapting the proof of Dumont \& Paoli (2006, Proposition 3.1).

LEMMA 4.1 Let $\beta=1 / 2$, then there exists a positive constant $C\left(f, u_{0}, v_{0}\right)$ depending only on the data, such that for all $h>0$ and for all $N \geqslant 1$

$$
\left|\frac{u_{n+1}^{h}-u_{n}^{h}}{\Delta t}\right|_{\mathbb{H}}^{2}+\frac{1}{2} a\left(u_{n}^{h}, u_{n}^{h}\right)+\frac{1}{2} a\left(u_{n+1}^{h}, u_{n+1}^{h}\right) \leqslant C\left(f, u_{0}, v_{0}\right)
$$

for $n \in\{1, \ldots, N-1\}$, where $\left(u_{n+1}^{h}\right)_{1 \leqslant n \leqslant N-1}$ are solutions of problem (4.1).

Now let us build the sequence of approximate solutions $\left(u_{h, N}\right)_{h>0, N \geqslant 1}$ of problem (4.1) by linear interpolation:

$$
\left\{\begin{array}{l}
\text { if } t \in[n \Delta t,(n+1) \Delta t], 0 \leqslant n \leqslant N-1, \text { we set } \\
u_{h, N}(x, t)=u_{n}^{h}(x) \frac{(n+1) \Delta t-t}{\Delta t}+u_{n+1}^{h}(x) \frac{t-n \Delta t}{\Delta t},
\end{array}\right.
$$

which is defined on $\Omega \times[0, T]$. Let us observe that these functions are continuous in time (obvious) and space (for all $n, u_{n}^{h}$ belongs to $H^{2}(\Omega)$, which is included in $\mathcal{C}^{0}(\bar{\Omega})$ ). Moreover, because of (4.2), for all $h>0$ and $N \geqslant 1$, the functions $u_{h, N}$ belong to $L^{\infty}(0, T ; \mathbb{V})$ and are uniformly bounded in this space. As $\dot{u}_{h, N}(x, t)=\frac{u_{n+1}^{h}(x)-u_{n}^{h}(x)}{\Delta t}$ for $t \in[n \Delta t,(n+1) \Delta t]$, using (4.2) again, functions $\dot{u}_{h, N}$ belong to $L^{\infty}\left(0, T ; L^{2}(\Omega)\right)$ and are also uniformly bounded in this space. So there exists a subsequence still denoted $\left(u_{h, N}\right)_{h>0, N \geqslant 1}$ and $u \in \mathbb{U}$ such that we have the following convergences:

$$
\begin{aligned}
& u_{h, N} \rightarrow u \text { weakly* in } L^{\infty}(0, T ; \mathbb{V}), \\
& \dot{u}_{h, N} \rightarrow \dot{u} \text { weakly* in } L^{\infty}\left(0, T ; L^{2}(\Omega)\right) .
\end{aligned}
$$

As the injection $H^{2}(\Omega) \hookrightarrow H^{1+\xi}(\Omega)$ is compact (Rellich's lemma, for $\xi<1$ ), and with Simon's lemma (Simon, 1987, Corollary 4, p. 85), we deduce that $\left\{w \in L^{\infty}(0, T ; \mathbb{V}), \dot{w} \in L^{\infty}\left(0, T ; L^{2}(\Omega)\right)\right\}$ is compactly embedded in $\mathcal{C}^{0}\left(0, T ; H^{1+\xi}(\Omega)\right)$ and then in $\mathcal{C}^{0}([0, T] \times \bar{\Omega})$. Therefore, after another subsequence extraction if necessary, we have

$$
u_{h, N} \rightarrow u \text { strongly in } \mathcal{C}^{0}\left(0, T ; H^{1+\xi}(\Omega)\right) \text { and in } \mathcal{C}^{0}([0, T] \times \bar{\Omega}) .
$$


Consequently, we obtain the following results.

- As $L^{\infty}\left(0, T ; L^{2}(\Omega)\right)$ is included in $L^{2}\left(0, T ; L^{2}(\Omega)\right)$, $\dot{u}$ belongs to this space. Moreover, as all functions $u_{h, N}$ belong to $L^{2}(0, T ; \mathbb{K}), u$ also belongs to it. So $u$ belongs to $\tilde{U}$.

- For every $h$ and $N, u_{h, N}(x, 0)=u_{0}^{h}(x)$, which converges towards $u_{0}$ in $\mathbb{V}$ (see (3.1)). As $\mathbb{V} \subset H^{1+\xi}(\Omega)$ with continuous injection, then $u(\cdot, 0)=u_{0}$.

Then we shall prove the following result.

THEOREM 4.2 Let $\beta=1 / 2$. Then the sequence of approximate solutions $\left(u_{h, N}\right)_{h>0, N \geqslant 1}$ given by (4.3) converges weakly* to $u$ in $\left\{w \in L^{\infty}(0, T ; \mathbb{V}) / \dot{w} \in L^{\infty}\left(0, T ; L^{2}(\Omega)\right)\right\}$. Moreover, $u$ belongs to $\tilde{\mathbb{U}}$, is such that $u(\cdot, 0)=u_{0}$ and is a solution of problem $(2.8)$.

The corollary is that the frictionless elastodynamic problem for a Kirchhoff-Love bending plate between two rigid obstacles has at least one solution.

Proof (Construction of a discrete test function.)

To obtain (2.8) from (4.1), a first point is to associate to any test function $\tilde{w}$ a discrete one which is close to it. A natural idea would be to define $w_{n}^{h}$ as the linear projection, defined by the bilinear form $a$, on the space $\mathbb{V}^{h}$, of an approximate value of $\tilde{w}$ at time $n \Delta t$. Unfortunately, this projection does not preserve the unilateral constraints. Then this choice would not necessarily give a test function in $\mathbb{K}^{h}$.

So, let $\tilde{w}$ be a test function such that $\tilde{w} \in \tilde{\mathbb{U}}$ and $\tilde{w}(\cdot, T)=u(\cdot, T)$. For $\varepsilon \in] 0, T / 2[$, we define $\phi$ as a $\mathcal{C}^{1}$ function such that

$$
\begin{cases}0 \leqslant \phi(t) \leqslant 1, & t \in[0, T] \\ \phi(t)=0, & t \in[T-3 \varepsilon / 2, T] \\ \phi(t)=1, & t \in[0, T-2 \varepsilon] .\end{cases}
$$

We set $w=(1-\phi) u+\phi \tilde{w}$. Then, by construction, $w(\cdot, t)=u(\cdot, t)$ for all $t \in[T-3 \varepsilon / 2, T]$. And, since $\mathbb{K}$ is convex we immediately have $w \in \tilde{\mathbb{U}}$.

Now let $\eta \in] 0, \varepsilon / 2[$ and $\chi \in] 0,1\left[\right.$. Following Dumont \& Paoli (2006), we define $w_{\eta, \chi}$ by

$$
w_{\eta, \chi}(x, t)=u(x, t)+\frac{1}{\eta} \int_{t}^{t+\eta}((1-\chi) w(x, s)-u(x, s)) \mathrm{d} s, t \in[0, T-\varepsilon / 2] .
$$

Since $u \in \mathbb{U}$ and $w \in \tilde{\mathbb{U}}$ we clearly have

$$
\left\{\begin{array}{l}
w_{\eta, \chi}-u \in \mathcal{C}^{0}(0, T-\varepsilon / 2 ; \mathbb{V}) \\
w_{\eta, \chi} \in L^{\infty}(0, T-\varepsilon / 2 ; \mathbb{V}) \cap \mathcal{C}^{0}\left(0, T-\varepsilon / 2 ; H^{1+\xi}(\Omega)\right), \\
\dot{w}_{\eta, \chi} \in L^{2}\left(0, T-\varepsilon / 2 ; L^{2}(\Omega)\right)
\end{array}\right.
$$

Moreover, we can select $\eta$ such that $w_{\eta, \chi}$ strictly satisfies the constraint. More precisely, for all $t \in[0, T$ $-\varepsilon / 2]$ and for all $x \in \Omega$,

$$
w_{\eta, \chi}(x, t)=\frac{1}{\eta} \int_{t}^{t+\eta}(1-\chi) w(x, s) \mathrm{d} s+u(x, t)-\frac{1}{\eta} \int_{t}^{t+\eta} u(x, s) \mathrm{d} s .
$$

Let us recall that, in the definition of the convex set $\mathbb{K}$, a scalar $g$ is introduced such that

$$
g_{1}(x) \leqslant-g<0<g \leqslant g_{2}(x) \quad \forall x \in \Omega .
$$


First, as $w \in \tilde{\mathbb{U}}$, we have $g_{1}(x) \leqslant w(x, t) \leqslant g_{2}(x)$ for all $x$ and $t$. So,

$$
g_{1}(x)+\chi g \leqslant(1-\chi) g_{1}(x) \leqslant \frac{1}{\eta} \int_{t}^{t+\eta}(1-\chi) w(x, s) \mathrm{d} s \leqslant(1-\chi) g_{2}(x) \leqslant g_{2}(x)-\chi g .
$$

Second, let us recall that $u$ belongs to $\mathcal{C}^{0}([0, T] \times \bar{\Omega})$. Thus, by uniform continuity on a compact set, for all $\delta$ belongs to ] $0, \chi g / 2[$ (constant $g>0$ is defined by (2.7)), there exists $\eta>0$ such that for all $x$, $|u(x, t)-u(x, s)|<\delta$ whenever $|t-s|<\eta$. Then

$$
\left|u(x, t)-\frac{1}{\eta} \int_{t}^{t+\eta} u(x, s) \mathrm{d} s\right| \leqslant \frac{1}{\eta} \int_{t}^{t+\eta}|u(x, t)-u(x, s)| \mathrm{d} s \leqslant \frac{1}{\eta} \eta \delta=\delta<\chi \frac{g}{2} .
$$

Finally, we have

$$
g_{1}(x)+\frac{\chi g}{2} \leqslant w_{\eta, \chi}(x, t) \leqslant g_{2}(x)-\frac{\chi g}{2} \quad \forall x \in \Omega, \forall t \in[0, T-\varepsilon / 2],
$$

which ensures that $w_{\eta, \chi}(x, t)$ belongs to $\left[g_{1}(x)+\chi g / 2, g_{2}(x)-\chi g / 2\right]$.

LEMMA 4.3 (Construction of a discrete test function.)

For $x$ belongs to $\Omega$, let $w_{n}^{h}$ be

$$
w_{n}^{h}(x)= \begin{cases}u_{n+1}^{h}(x)+\pi_{h}\left(w_{\eta, \chi}(x, n \Delta t)-u(x, n \Delta t)\right) & \text { if } n \Delta t \leqslant T-\varepsilon, \\ u_{n+1}^{h}(x) & \text { if } n \Delta t>T-\varepsilon,\end{cases}
$$

where $\pi_{h}$ is linear projection, defined by the bilinear form $a$, on space $\mathbb{V}^{h}$. Then there exists $h_{0}>$ 0 and $N_{0} \geqslant 1$ such that, for all $\left.h \in\right] 0, h_{0}\left[\right.$ and for all $N \geqslant N_{0}, w_{n}^{h}$ belongs to $\mathbb{K}^{h}$ for all $n$ belong to $\{1, \ldots, N-1\}$.

\section{Proof}

- It is obvious that $w_{n}^{h}$ belongs to $\mathbb{V}^{h}$ and $\mathbb{K}^{h}$ when $n \Delta t>T-\varepsilon$.

- Otherwise, when $n \Delta t \leqslant T-\varepsilon, w_{n}^{h}$ is written as

$$
\begin{aligned}
w_{n}^{h}(x)= & u_{h, N}(x,(n+1) \Delta t)-u(x,(n+1) \Delta t) \\
& +u(x,(n+1) \Delta t)-u(x, n \Delta t)+w_{\eta, \chi}(x, n \Delta t) \\
& +\left(\pi_{h}-I d\right)\left(w_{\eta, \chi}(x, n \Delta t)-u(x, n \Delta t)\right) .
\end{aligned}
$$

First, as $\left(u_{h, N}\right)_{h>0, N \geqslant 1}$ converges strongly to $u$ in $\mathcal{C}^{0}\left(0, T ; H^{1+\xi}(\Omega)\right)$, and using the continuity of the canonical injection from $H^{1+\xi}(\Omega)$ into $\mathcal{C}^{0}(\bar{\Omega})$, for $h$ small enough and $N$ large enough, we obtain

$$
\sup _{x \in \bar{\Omega}}\left|u_{h, N}(x,(n+1) \Delta t)-u(x,(n+1) \Delta t)\right| \leqslant C\left\|u_{h, N}-u\right\|_{\mathcal{C}^{0}\left(0, T ; H^{1+\xi}(\Omega)\right)} \leqslant \frac{\chi g}{6} .
$$

Second, $u$ is continuous on the compact set $[0, T] \times \bar{\Omega}$. So, by uniform continuity, there exists $\Delta t_{0}$ or $N_{0}=T / \Delta t_{0}$, such that if $\Delta t \leqslant \Delta t_{0}$ or $N \geqslant N_{0}$, we have

$$
\sup _{x \in \bar{\Omega}}|u(x,(n+1) \Delta t)-u(x, n \Delta t)| \leqslant \frac{\chi g}{6} .
$$


Third, let us introduce the constant $\gamma_{h}$, which depends on $h$ : because of the canonical embedding from $\mathbb{V}$ to $H^{1+\xi}(\Omega)$ and the convergence of the finite element scheme, for all $h>0$, there exists $\gamma_{h}$ such that

$$
\forall w \in \mathbb{V},\left\|\pi_{h} w-w\right\|_{H^{1+\xi}(\Omega)} \leqslant \gamma_{h}\|w\|_{\mathbb{V}} \text { and } \lim _{h \rightarrow 0} \gamma_{h}=0
$$

Then, for $h$ small enough,

$$
\begin{aligned}
\sup _{x \in \bar{\Omega}}\left|\left(\pi_{h}-I d\right)\left(w_{\eta, \chi}(x, n \Delta t)-u(x, n \Delta t)\right)\right| & \leqslant C\left\|\left(\pi_{h}-I d\right)\left(w_{\eta, \chi}(\cdot, n \Delta t)-u(\cdot, n \Delta t)\right)\right\|_{H^{1+\xi}(\Omega)} \\
& \leqslant C \gamma_{h}\left\|w_{\eta, \chi}-u\right\|_{L^{\infty}(0, T-\varepsilon / 2 ; \mathbb{V})} \leqslant \frac{\chi g}{6} .
\end{aligned}
$$

Finally, using the previous results, for $h$ small enough and $N$ large enough, we have

$$
-\frac{\chi g}{2} \leqslant w_{n}^{h}(x)-w_{\eta, \chi}(x, n \Delta t) \leqslant \frac{\chi g}{2}
$$

for all $x \in \Omega$ and $n \in\{1, \ldots, N-1\}$, which leads to

$$
g_{1}(x) \leqslant w_{\eta, \chi}(x, n \Delta t)-\frac{\chi g}{2} \leqslant w_{n}^{h}(x) \leqslant w_{\eta, \chi}(x, n \Delta t)+\frac{\chi g}{2} \leqslant g_{2}(x),
$$

by using (4.6). And we can conclude that $w_{n}^{h}$ belongs to $\mathbb{K}^{h}$.

\subsection{Transformation of inequality}

Now, our goal is to show that the limit $u$ is a solution of the continuous impact problem (2.8). So, to use the previous lemma, in all the following we will assume that $h \in] 0, h_{0}\left[\right.$ and $N \geqslant N_{0}$. Thus, we set $\Delta t=T / N$. In (4.1) we take $w^{h}=w_{n}^{h}$, we multiply by $\Delta t$ and sum over $n$ to obtain

$$
\begin{aligned}
& \sum_{n=1}^{N-1}\left(\int_{\Omega} \frac{u_{n+1}^{h}-2 u_{n}^{h}+u_{n-1}^{h}}{(\Delta t)^{2}}\left(w_{n}^{h}-u_{n+1}^{h}\right) \mathrm{d} x\right) \Delta t+\sum_{n=1}^{N-1}\left(\frac{1}{2} a\left(u_{n+1}^{h}+u_{n-1}^{h}, w_{n}^{h}-u_{n+1}^{h}\right)\right) \Delta t \\
& \quad \geqslant \sum_{n=1}^{N-1}\left(\frac{1}{2} \int_{\Omega}\left(f_{n+1}+f_{n-1}\right)\left(w_{n}^{h}-u_{n+1}^{h}\right) \mathrm{d} x\right) \Delta t
\end{aligned}
$$

From the definition of the discrete test function (Lemma 4.3), we have $w_{n}^{h}-u_{n+1}^{h}=0$ as far as $n \Delta t>$ $T-\varepsilon$. So the above sums end at the integer $N^{\prime}$, which is the integer part of $\frac{(T-\varepsilon)}{\Delta t}$. 
4.8) can be rewritten as

$$
\begin{aligned}
\sum_{n=1}^{N^{\prime}} & \int_{\Omega} \frac{u_{n+1}^{h}-2 u_{n}^{h}+u_{n-1}^{h}}{(\Delta t)^{2}}\left(w_{n}^{h}-u_{n+1}^{h}\right) \mathrm{d} x \\
= & \sum_{n=1}^{N^{\prime}} \int_{\Omega}\left(\frac{u_{n+1}^{h}-u_{n}^{h}}{\Delta t}-\frac{u_{n}^{h}-u_{n-1}^{h}}{\Delta t}\right) \frac{w_{n}^{h}-u_{n+1}^{h}}{\Delta t} \mathrm{~d} x \\
= & \int_{\Omega} \frac{u_{N^{\prime}+1}^{h}-u_{N^{\prime}}^{h}}{\Delta t} \frac{w_{N^{\prime}}^{h}-u_{N^{\prime}+1}^{h}}{\Delta t} \mathrm{~d} x-\sum_{n=1}^{N^{\prime}} \int_{\Omega} \frac{u_{n}^{h}-u_{n-1}^{h}}{\Delta t} \frac{\left(w_{n}^{h}-u_{n+1}^{h}\right)-\left(w_{n-1}^{h}-u_{n}^{h}\right)}{\Delta t} \mathrm{~d} x \\
& -\int_{\Omega} \frac{u_{1}^{h}-u_{0}^{h}}{\Delta t} \frac{w_{0}^{h}-u_{1}^{h}}{\Delta t} \mathrm{~d} x \\
= & -\sum_{n=1}^{N^{\prime}+1} \int_{\Omega} \frac{u_{n}^{h}-u_{n-1}^{h}}{\Delta t} \frac{\left(w_{n}^{h}-u_{n+1}^{h}\right)-\left(w_{n-1}^{h}-u_{n}^{h}\right)}{\Delta t} \mathrm{~d} x-\int_{\Omega} \frac{u_{1}^{h}-u_{0}^{h}}{\Delta t} \frac{w_{0}^{h}-u_{1}^{h}}{\Delta t} \mathrm{~d} x
\end{aligned}
$$

as $w_{N^{\prime}+1}^{h}-u_{N^{\prime}+2}^{h}=0$. Finally, we have

$$
\begin{aligned}
\int_{\Omega} & \frac{u_{1}^{h}-u_{0}^{h}}{\Delta t}\left(w_{0}^{h}-u_{1}^{h}\right) \mathrm{d} x+\sum_{n=1}^{N^{\prime}}\left(\frac{1}{2} \int_{\Omega}\left(f_{n+1}+f_{n-1}\right)\left(w_{n}^{h}-u_{n+1}^{h}\right) \mathrm{d} x\right) \Delta t \\
\leqslant & \sum_{n=1}^{N^{\prime}}\left(\frac{1}{2} a\left(u_{n+1}^{h}+u_{n-1}^{h}, w_{n}^{h}-u_{n+1}^{h}\right)\right) \Delta t \\
& -\sum_{n=1}^{N^{\prime}+1}\left(\int_{\Omega} \frac{u_{n}^{h}-u_{n-1}^{h}}{\Delta t} \frac{\left(w_{n}^{h}-u_{n+1}^{h}\right)-\left(w_{n-1}^{h}-u_{n}^{h}\right)}{\Delta t} \mathrm{~d} x\right) \Delta t .
\end{aligned}
$$

The goal of the remainder of this proof is to make $h$ and $\Delta t$ tend to zero. So each term of the previous expression will be examined separately in the four following steps.

Step 1. By definition, $w_{0}^{h}(x)-u_{1}^{h}(x)=\pi_{h}\left(w_{\eta, \chi}(x, 0)-u(x, 0)\right)$. Then

$$
\begin{aligned}
\int_{\Omega} \frac{u_{1}^{h}-u_{0}^{h}}{\Delta t}\left(w_{0}^{h}-u_{1}^{h}\right) \mathrm{d} x= & \int_{\Omega} \frac{u_{1}^{h}-u_{0}^{h}}{\Delta t}\left(\pi_{h}-I d\right)\left(w_{\eta, \chi}(x, 0)-u(x, 0)\right) \mathrm{d} x \\
& +\int_{\Omega} \frac{u_{1}^{h}-u_{0}^{h}}{\Delta t}\left(w_{\eta, \chi}(x, 0)-u(x, 0)\right) \mathrm{d} x .
\end{aligned}
$$

So, (4.7) leads to

$$
\begin{aligned}
\left|\left(\pi_{h}-I d\right)\left(w_{\eta, \chi}(\cdot, 0)-u(\cdot, 0)\right)\right|_{\mathbb{H}} & \leqslant\left\|\left(\pi_{h}-I d\right)\left(w_{\eta, \chi}(\cdot, 0)-u(\cdot, 0)\right)\right\|_{H^{1+\xi}(\Omega)} \\
& \leqslant \gamma_{h}\left\|\left(w_{\eta, \chi}(\cdot, 0)-u(\cdot, 0)\right)\right\|_{\mathbb{V}},
\end{aligned}
$$

with $\lim _{h \rightarrow 0} \gamma_{h}=0$. Finally, from (3.1), it is known that $\lim _{h \rightarrow 0, \Delta t \rightarrow 0}\left|\frac{u_{1}^{h}-u_{0}^{h}}{\Delta t}-v_{0}\right|_{\mathbb{H}}=0$, and we obtain 


$$
\int_{\Omega} \frac{u_{1}^{h}-u_{0}^{h}}{\Delta t}\left(w_{0}^{h}-u_{1}^{h}\right) \mathrm{d} x \stackrel{h, \Delta t}{\longrightarrow} 0 \quad \int_{\Omega} v_{0}(x)\left(w_{\eta, \chi}(x, 0)-u(x, 0)\right) \mathrm{d} x .
$$

Step 2. The second term of (4.9) can be split in two parts of the same following form:

$$
\begin{aligned}
\sum_{n=1}^{N^{\prime}} \int_{\Omega} f_{n^{\prime}}\left(w_{n}^{h}-u_{n+1}^{h}\right) \mathrm{d} x \Delta t= & \sum_{n=1}^{N^{\prime}} \int_{\Omega} f_{n^{\prime}}(x) \pi_{h}\left(w_{\eta, \chi}(x, n \Delta t)-u(x, n \Delta t)\right) \mathrm{d} x \Delta t \\
= & \sum_{n=1}^{N^{\prime}} \int_{\Omega} f_{n^{\prime}}(x)\left(\pi_{h}-I d\right)\left(w_{\eta, \chi}(x, n \Delta t)-u(x, n \Delta t)\right) \mathrm{d} x \Delta t \\
& +\sum_{n=1}^{N^{\prime}} \int_{n^{\prime} \Delta t}^{\left(n^{\prime}+1\right) \Delta t} \int_{\Omega} f(x, s)\left[\left(w_{\eta, \chi}(x, n \Delta t)-u(x, n \Delta t)\right)\right. \\
& \left.+\sum_{n=1}^{N^{\prime}} \int_{n^{\prime} \Delta t}^{\left(n^{\prime}+1\right) \Delta t} \int_{\Omega} f(x, s)\left(w_{\eta, \chi}(x, s)-u(x, s)\right)\right] \mathrm{d} x \mathrm{~d} s \\
\equiv & S_{1}+S_{2}+S_{3}
\end{aligned}
$$

from the definition of $f_{n^{\prime}}$ (see (3.2)), and those of the discrete test function $w_{n}^{h}$. Here we have $n^{\prime}=n+1$ or $n^{\prime}=n-1$. Let us examine each of these terms successively.

(1) As in Step 1, (4.7) leads to

$$
\begin{aligned}
\left|\left(\pi_{h}-I d\right)\left(w_{\eta, \chi}(\cdot, n \Delta t)-u(\cdot, n \Delta t)\right)\right|_{\mathbb{H}} & \leqslant \gamma_{h}\left\|\left(w_{\eta, \chi}(\cdot, n \Delta t)-u(\cdot, n \Delta t)\right)\right\|_{\mathbb{V}} \\
& \leqslant \gamma_{h}\left\|w_{\eta, \chi}-u\right\|_{L^{\infty}(0, T-\varepsilon / 2 ; \mathbb{V})}
\end{aligned}
$$

for all $n \in\left\{1, \ldots, N^{\prime}\right\}$. Then we deduce that

$$
\begin{aligned}
\left|S_{1}\right| & =\left|\sum_{n=1}^{N^{\prime}} \int_{\Omega} f_{n^{\prime}}(x)\left(\pi_{h}-I d\right)\left(w_{\eta, \chi}(x, n \Delta t)-u(x, n \Delta t)\right) \mathrm{d} x \Delta t\right| \\
& \leqslant\left(\sum_{n=1}^{N^{\prime}} \mid f_{n^{\prime}} \mathbb{H} \Delta t\right) \gamma_{h}\left\|w_{\eta, \chi}-u\right\|_{L^{\infty}(0, T-\varepsilon / 2 ; \mathbb{V})} \\
& \leqslant\left(\sqrt{T}\|f\|_{L^{2}(0, T ; \mathbb{H})}\right) \gamma_{h}\left\|w_{\eta, \chi}-u\right\|_{L^{\infty}(0, T-\varepsilon / 2 ; \mathbb{V})} \stackrel{h \rightarrow 0}{\longrightarrow} 0 .
\end{aligned}
$$

(2) The definition of $w_{\eta, \chi},(4.5)$, leads to

$$
\begin{aligned}
& \left(w_{\eta, \chi}(x, n \Delta t)-u(x, n \Delta t)\right)-\left(w_{\eta, \chi}(x, s)-u(x, s)\right) \\
& \quad=\frac{1}{\eta} \int_{n \Delta t}^{n \Delta t+\eta}((1-\chi) w(x, t)-u(x, t)) \mathrm{d} t-\frac{1}{\eta} \int_{s}^{s+\eta}((1-\chi) w(x, t)-u(x, t)) \mathrm{d} t \\
& =\frac{1}{\eta} \int_{n \Delta t}^{s}((1-\chi) w(x, t)-u(x, t)) \mathrm{d} t-\frac{1}{\eta} \int_{n \Delta t+\eta}^{s+\eta}((1-\chi) w(x, t)-u(x, t)) \mathrm{d} t .
\end{aligned}
$$


Moreover, if $\varphi$ belongs to $L^{2}(0, T ; \mathbb{H}), a$ and $b$ being such that $0 \leqslant a<b \leqslant T$, one has

$$
\begin{aligned}
\left|\int_{a}^{b} \varphi(\cdot, t) \mathrm{d} t\right|_{\mathbb{H}}^{2} & =\int_{\Omega}\left(\int_{a}^{b} \varphi(x, t) \mathrm{d} t\right)^{2} \mathrm{~d} x \\
& \leqslant(b-a) \int_{\Omega} \int_{a}^{b} \varphi^{2}(x, t) \mathrm{d} t \mathrm{~d} x \leqslant(b-a)\|\varphi\|_{L^{2}(0, T ; \mathbb{H})}^{2}
\end{aligned}
$$

or else

$$
\left|\int_{a}^{b} \varphi(\cdot, t) \mathrm{d} t\right|_{\mathbb{H}} \leqslant \sqrt{b-a}\|\varphi\|_{L^{2}(0, T ; \mathbb{H})} .
$$

This result implies that

$$
\begin{aligned}
& \left|\left(w_{\eta, \chi}(\cdot, n \Delta t)-u(\cdot, n \Delta t)\right)-\left(w_{\eta, \chi}(\cdot, s)-u(\cdot, s)\right)\right|_{\mathbb{H}} \\
& \quad \leqslant \frac{2 \sqrt{|s-n \Delta t|}}{\eta}\|(1-\chi) w-u\|_{L^{2}(0, T ; \mathbb{H})} \\
& \quad \leqslant \frac{2 \sqrt{|s-n \Delta t|}}{\eta}\|(1-\chi) w-u\|_{L^{2}(0, T ; \mathbb{V})} .
\end{aligned}
$$

As $s$ belongs to $[(n-1) \Delta t, n \Delta t]$ or $[(n+1) \Delta t,(n+2) \Delta t]$, in all cases we obtain

$$
\begin{aligned}
\left|S_{2}\right|=\sum_{n=1}^{N^{\prime}} \int_{n^{\prime} \Delta t}^{\left(n^{\prime}+1\right) \Delta t} \int_{\Omega} f(x, s)\left[\left(w_{\eta, \chi}(x, n \Delta t)-u(x, n \Delta t)\right)\right. \\
\left.-\left(w_{\eta, \chi}(x, s)-u(x, s)\right)\right] \mathrm{d} x \mathrm{~d} s \\
\leqslant\left(\sum_{n=1}^{N^{\prime}} \int_{n^{\prime} \Delta t}^{\left(n^{\prime}+1\right) \Delta t}|f(\cdot, s)|_{\mathbb{H}} \mathrm{d} s\right) \frac{2 \sqrt{2 \Delta t}}{\eta}\|(1-\chi) w-u\|_{L^{2}(0, T ; \mathbb{V})} \\
\leqslant \sqrt{T}\|f\|_{L^{2}(0, T ; \mathbb{H})} \frac{2 \sqrt{2 \Delta t}}{\eta}\|(1-\chi) w-u\|_{L^{2}(0, T ; \mathbb{V}) \stackrel{\Delta t \rightarrow 0}{\rightarrow} 0 .}
\end{aligned}
$$

(3) Finally, as $w_{\eta, \chi}-u$ belongs to $\mathcal{C}^{0}(0, T-\varepsilon / 2 ; \mathbb{V})$, which is contained in $L^{2}(0, T ; \mathbb{H})$, as $f$ is in $L^{2}(0, T ; \mathbb{H})$ and $N^{\prime}$ is the integer part of $\frac{T-\varepsilon}{\Delta t}$, then we can make $\Delta t$ go to zero and obtain

$$
\begin{aligned}
& S_{3}=\sum_{n=1}^{N^{\prime}} \int_{n^{\prime} \Delta t}^{\left(n^{\prime}+1\right) \Delta t} \int_{\Omega} f(x, s)\left(w_{\eta, \chi}(x, s)-u(x, s)\right) \mathrm{d} x \mathrm{~d} s \\
&= \begin{cases}\int_{2 \Delta t}^{\left(N^{\prime}+2\right) \Delta t} \int_{\Omega} f(x, s)\left(w_{\eta, \chi}(x, s)-u(x, s)\right) \mathrm{d} x \mathrm{~d} s & \text { if } n^{\prime}=n+1 \\
\int_{0}^{N^{\prime} \Delta t} \int_{\Omega} f(x, s)\left(w_{\eta, \chi}(x, s)-u(x, s)\right) \mathrm{d} x \mathrm{~d} s & \text { if } n^{\prime}=n-1\end{cases} \\
& \stackrel{\Delta t}{\longrightarrow} 0 \int_{0}^{T-\varepsilon} \int_{\Omega} f(x, s)\left(w_{\eta, \chi}(x, s)-u(x, s)\right) \mathrm{d} x \mathrm{~d} s .
\end{aligned}
$$


So, we can conclude this step and have

$$
\begin{gathered}
\sum_{n=1}^{N^{\prime}}\left(\frac{1}{2} \int_{\Omega}\left(f_{n+1}+f_{n-1}\right)\left(w_{n}^{h}-u_{n+1}^{h}\right) \mathrm{d} x\right) \Delta t \\
\quad h, \stackrel{\Delta t}{\longrightarrow} 0 \int_{0}^{T-\varepsilon} \int_{\Omega} f\left(w_{\eta, \chi}-u\right) \mathrm{d} x \mathrm{~d} s
\end{gathered}
$$

Step 3. We carry on with the convergence of the third term of (4.9). Here we shall use some results which we will reuse later.

- The bilinear form $a$ defines a scalar product on $\mathbb{V}$, which is equivalent to the canonical scalar product (see Lemma 2.1). So there exists $C>0$ such that $|a(w, w)| \leqslant C\|w\|_{\mathbb{V}}$ for all $w \in \mathbb{V}$.

- $\pi_{h}$ is the linear projection on the space $\mathbb{V}^{h}$ defined by the bilinear form $a$. In particular, for all $w^{h} \in \mathbb{V}^{h}$ and $v \in \mathbb{V}, a\left(w^{h}, \pi_{h} v\right)=a\left(w^{h}, v\right)$.

Now, let us observe that

$$
\begin{aligned}
\frac{1}{2} & \sum_{n=1}^{N^{\prime}} a\left(u_{n+1}^{h}+u_{n-1}^{h}, w_{n}^{h}-u_{n+1}^{h}\right) \Delta t \\
= & \frac{1}{2} a\left(u_{0}^{h}, w_{0}^{h}-u_{1}^{h}\right) \Delta t+\frac{1}{2} \sum_{n=1}^{N^{\prime}+1} a\left(u_{n-1}^{h},\left(w_{n}^{h}-u_{n+1}^{h}\right)-\left(w_{n-1}^{h}-u_{n}^{h}\right)\right) \Delta t \\
& +\frac{1}{2} \sum_{n=1}^{N^{\prime}} a\left(u_{n+1}^{h}+u_{n}^{h}, w_{n}^{h}-u_{n+1}^{h}\right) \Delta t \\
\equiv & \frac{1}{2} S_{1}+\frac{1}{2} S_{2}+S_{3},
\end{aligned}
$$

as $w_{N^{\prime}+1}^{h}-u_{N^{\prime}+2}^{h}=0$. Now each of these terms will be studied.

(1) By definition, $w_{0}^{h}(x)-u_{1}^{h}(x)=\pi_{h}\left(w_{\eta, \chi}(x, 0)-u(x, 0)\right)$. So

$$
\begin{aligned}
\left|S_{1}\right| & =\left|a\left(u_{0}^{h}, w_{0}^{h}-u_{1}^{h}\right)\right| \Delta t=\mid a\left(u_{0}^{h}, w_{\eta, \chi}(\cdot, 0)-u(\cdot, 0) \mid \Delta t\right. \\
& \leqslant C^{2}\left\|u_{0}^{h}\right\|_{\mathbb{V}}\left\|w_{\eta, \chi}(\cdot, 0)-u(\cdot, 0)\right\|_{\mathbb{V}} \Delta t \stackrel{h, \Delta t \rightarrow 0}{\longrightarrow} 0,
\end{aligned}
$$

$\left(u_{0}^{h}\right)_{h}$ being bounded as the time scheme is initialized by choosing $u_{0}^{h}$ such that $\lim _{h \rightarrow 0}\left\|u_{0}^{h}-u_{0}\right\|_{\mathbb{V}}=0$ (see $\left.(3.1)\right)$.

(2) Here again, from the definitions of the test functions $w_{p}^{h}$ and the projection $\pi_{h}$, we have

$$
\begin{aligned}
S_{2} & =\sum_{n=1}^{N^{\prime}+1} a\left(u_{n-1}^{h},\left(w_{n}^{h}-u_{n+1}^{h}\right)-\left(w_{n-1}^{h}-u_{n}^{h}\right)\right) \Delta t \\
& =\sum_{n=1}^{N^{\prime}+1} a\left(u_{n-1}^{h},\left(w_{\eta, \chi}-u\right)(\cdot, n \Delta t)-\left(w_{\eta, \chi}-u\right)(\cdot,(n-1) \Delta t)\right) \Delta t .
\end{aligned}
$$


Following Step $2(2)$, with $s=(n-1) \Delta t$, we obtain

$\left|\left(w_{\eta, \chi}-u\right)(\cdot, n \Delta t)-\left(w_{\eta, \chi}-u\right)(\cdot,(n-1) \Delta t)\right|_{\mathbb{H}} \leqslant \frac{2 \sqrt{\Delta t}}{\eta}\|(1-\chi) w-u\|_{L^{2}(0, T ; \mathbb{H})}$.

This property can be extended to the space derivatives (in the distribution sense) of $(1-\chi) w-u$ exactly in the same way and leads to

$$
\begin{aligned}
& \left\|\left(w_{\eta, \chi}-u\right)(\cdot, n \Delta t)-\left(w_{\eta, \chi}-u\right)(\cdot,(n-1) \Delta t)\right\|_{\mathbb{V}} \\
& \quad \leqslant \frac{2 \sqrt{\Delta t}}{\eta}\|(1-\chi) w-u\|_{L^{2}(0, T ; \mathbb{V})} .
\end{aligned}
$$

Then, using this inequality and (4.2), we have

$$
\begin{aligned}
\left|S_{2}\right| & \leqslant \sum_{n=1}^{N^{\prime}+1} \sqrt{a\left(u_{n-1}^{h}, u_{n-1}^{h}\right)} \frac{2 C \sqrt{\Delta t}}{\eta}\|(1-\chi) w-u\|_{L^{2}(0, T ; \mathbb{V})} \Delta t \\
& \leqslant \sum_{n=1}^{N^{\prime}+1} \sqrt{2 C\left(f, u_{0}, v_{0}\right)} \frac{2 C \sqrt{\Delta t}}{\eta}\|(1-\chi) w-u\|_{L^{2}(0, T ; \mathbb{V})} \Delta t \\
& \leqslant T \sqrt{2 C\left(f, u_{0}, v_{0}\right)} \frac{2 C \sqrt{\Delta t}}{\eta}\|(1-\chi) w-u\|_{L^{2}(0, T ; \mathbb{V})} \stackrel{\Delta t \rightarrow 0}{\rightarrow} 0 .
\end{aligned}
$$

(3) As the function $u_{h, N}$ is linear in time on each interval $[n \Delta t,(n+1) \Delta t]$ (see (4.3)), we have

$$
\int_{n \Delta t}^{(n+1) \Delta t} u_{h, N}(\cdot, s) \mathrm{d} s=\frac{1}{2}\left(u_{n+1}^{h}+u_{n}^{h}\right) \Delta t,
$$

which allows the third term to be rewritten as

$$
\begin{aligned}
S_{3}= & \frac{1}{2} \sum_{n=1}^{N^{\prime}} a\left(u_{n+1}^{h}+u_{n}^{h}, w_{n}^{h}-u_{n+1}^{h}\right) \Delta t=\sum_{n=1}^{N^{\prime}} \int_{n \Delta t}^{(n+1) \Delta t} a\left(u_{h, N}(\cdot, s), w_{n}^{h}-u_{n+1}^{h}\right) \mathrm{d} s \\
= & \sum_{n=1}^{N^{\prime}} \int_{n \Delta t}^{(n+1) \Delta t} a\left(u_{h, N}(\cdot, s),\left(w_{\eta, \chi}-u\right)(\cdot, n \Delta t)\right) \mathrm{d} s \\
= & \sum_{n=1}^{N^{\prime}} \int_{n \Delta t}^{(n+1) \Delta t} a\left(u_{h, N}(\cdot, s),\left(w_{\eta, \chi}-u\right)(\cdot, n \Delta t)-\left(w_{\eta, \chi}-u\right)(\cdot, s)\right) \mathrm{d} s \\
& +\int_{0}^{T-\varepsilon} a\left(u_{h, N}(\cdot, s),\left(w_{\eta, \chi}-u\right)(\cdot, s)\right) \mathrm{d} s-\int_{0}^{\Delta t} a\left(u_{h, N}(\cdot, s),\left(w_{\eta, \chi}-u\right)(\cdot, s)\right) \mathrm{d} s \\
& -\int_{\left(N^{\prime}+1\right) \Delta t}^{T-\varepsilon} a\left(u_{h, N}(\cdot, s),\left(w_{\eta, \chi}-u\right)(\cdot, s)\right) \mathrm{d} s .
\end{aligned}
$$


Replacing $(n-1) \Delta t$ in (4.12) by $s$, where $s$ belongs to $[n \Delta t,(n+1) \Delta t]$, we obtain

$$
\begin{aligned}
& \sum_{n=1}^{N^{\prime}} \int_{n \Delta t}^{(n+1) \Delta t} a\left(u_{h, N}(\cdot, s),\left(w_{\eta, \chi}-u\right)(\cdot, n \Delta t)-\left(w_{\eta, \chi}-u\right)(\cdot, s)\right) \mathrm{d} s \\
& \quad \leqslant C^{2} T\left\|u_{h, N}\right\|_{L^{\infty}(0, T ; \mathbb{V})} \frac{2 \sqrt{\Delta t}}{\eta}\|(1-\chi) w-u\|_{L^{2}(0, T ; \mathbb{V})} \stackrel{\Delta t \rightarrow 0}{\longrightarrow} 0
\end{aligned}
$$

as functions $\left(u_{h, N}\right)_{h>0, N \geqslant 1}$ are uniformly bounded because of (4.2). The same reason leads to

$$
\begin{aligned}
& \left|\int_{0}^{\Delta t} a\left(u_{h, N}(\cdot, s),\left(w_{\eta, \chi}-u\right)(\cdot, s)\right) \mathrm{d} s\right| \\
& \quad \leqslant \int_{0}^{\Delta t} C^{2}\left\|u_{h, N}(\cdot, s)\right\| \mathbb{V}\left\|\left(w_{\eta, \chi}-u\right)(\cdot, s)\right\|_{\mathbb{V}} \mathrm{d} s \\
& \quad \leqslant \Delta t C^{2}\left\|u_{h, N}\right\|_{L^{\infty}(0, T ; \mathbb{V})}\left\|\left(w_{\eta, \chi}-u\right)\right\|_{L^{\infty}(0, T-\varepsilon / 2 ; \mathbb{V})} \\
& \quad \stackrel{\Delta t \rightarrow 0}{\longrightarrow} 0,
\end{aligned}
$$

and, in a similar way,

$$
\int_{\left(N^{\prime}+1\right) \Delta t}^{T-\varepsilon} a\left(u_{h, N}(\cdot, s),\left(w_{\eta, \chi}-u\right)(\cdot, s)\right) \mathrm{d} s \stackrel{\Delta t \rightarrow 0}{\longrightarrow} 0 .
$$

Finally, as the inclusion of $L^{\infty}(0, T ; \mathbb{V})$ into $L^{2}(0, T ; \mathbb{V})$ is continuous, functions $\left(u_{h, N}\right)_{h>0, N \geqslant 1}$, being uniformly bounded in $L^{\infty}(0, T ; \mathbb{V})$, are also uniformly bounded in $L^{2}(0, T ; \mathbb{V})$. So, up to a possible subsequence extraction, $\left(u_{h, N}\right)_{h>0, N \geqslant 1}$ converges weakly in this space towards $u$ (uniqueness of the limit). So that we obtain

$$
\int_{0}^{T-\varepsilon} a\left(u_{h, N}(\cdot, s),\left(w_{\eta, \chi}-u\right)(\cdot, s)\right) \mathrm{d} s \stackrel{h, \Delta t \rightarrow 0}{\longrightarrow} \int_{0}^{T-\varepsilon} a\left(u(\cdot, s),\left(w_{\eta, \chi}-u\right)(\cdot, s)\right) \mathrm{d} s
$$

and then

$$
\frac{1}{2} \sum_{n=1}^{N^{\prime}} a\left(u_{n+1}^{h}+u_{n-1}^{h}, w_{n}^{h}-u_{n+1}^{h}\right) \Delta t \stackrel{h, \Delta t \rightarrow 0}{\longrightarrow} \int_{0}^{T-\varepsilon} a\left(u(\cdot, s),\left(w_{\eta, \chi}-u\right)(\cdot, s)\right) \mathrm{d} s .
$$

Step 4. Finally, let us study the convergence of the fourth term of (4.9). To simplify the presentation, we introduce the notation

$$
\psi_{\Delta t}(x, t)=\frac{\left(w_{\eta, \chi}-u\right)(x, t+\Delta t)-\left(w_{\eta, \chi}-u\right)(x, t)}{\Delta t} \quad \forall t \in[0, T-\varepsilon / 2], \quad \forall x \in \Omega,
$$

and we recall that, by the definition of $N^{\prime}, w_{N^{\prime}+1}^{h}-u_{N^{\prime}+2}^{h}=0$ and that, by the definition of the discrete test functions (see Lemma 4.3), $w_{p}^{h}(x)-u_{p+1}^{h}(x)=\pi_{h}\left(w_{\eta, \chi}(x, p \Delta t)-u(x, p \Delta t)\right.$ ). 


$$
\begin{aligned}
& \left.\frac{1}{\Delta t} \frac{\left(w_{n}^{h}-u_{n+1}^{h}\right)-\left(w_{n-1}^{h}-u_{n}^{h}\right)}{\Delta t} \mathrm{~d} x\right) \Delta t \\
= & -\int_{\Omega} \frac{u_{N^{\prime}+1}^{h}-u_{N^{\prime}}^{h}}{\Delta t}\left(w_{N^{\prime}}^{h}-u_{N^{\prime}+1}^{h}\right) \mathrm{d} x \\
& +\sum_{n=1}^{N^{\prime}}\left(\int_{\Omega} \frac{u_{n}^{h}-u_{n-1}^{h}}{\Delta t}\left(\pi_{h}-I d\right) \psi_{\Delta t}(\cdot,(n-1) \Delta t) \mathrm{d} x\right) \Delta t \\
& +\sum_{n=1}^{N^{\prime}} \int_{(n-1) \Delta t}^{n \Delta t} \int_{\Omega} \frac{u_{n}^{h}-u_{n-1}^{h}}{\Delta t}\left(\psi_{\Delta t}(\cdot,(n-1) \Delta t)-\psi_{\Delta t}(\cdot, t)\right) \mathrm{d} x \mathrm{~d} t \\
& +\sum_{n=1}^{N^{\prime}} \int_{(n-1) \Delta t}^{n \Delta t} \int_{\Omega} \frac{u_{n}^{h}-u_{n-1}^{h}}{\Delta t} \psi_{\Delta t}(\cdot, t) \mathrm{d} x \mathrm{~d} t \\
\equiv & S_{1}+S_{2}+S_{3}+S_{4} .
\end{aligned}
$$

(1) First, using (4.2) and the definition of $w_{N^{\prime}}^{h}$, we have

$$
\begin{aligned}
\left|S_{1}\right|= & \left|\int_{\Omega} \frac{u_{N^{\prime}+1}^{h}-u_{N^{\prime}}^{h}}{\Delta t}\left(w_{N^{\prime}}^{h}-u_{N^{\prime}+1}^{h}\right) \mathrm{d} x\right| \\
= & \left|\int_{\Omega} \frac{u_{N^{\prime}+1}^{h}-u_{N^{\prime}}^{h}}{\Delta t} \pi_{h}\left(w_{\eta, \chi}\left(x, N^{\prime} \Delta t\right)-u\left(x, N^{\prime} \Delta t\right)\right) \mathrm{d} x\right| \\
\leqslant & \left|\frac{u_{N^{\prime}+1}^{h}-u_{N^{\prime}}^{h}}{\Delta t}\right|_{\mathbb{H}}\left|\pi_{h}\left(w_{\eta, \chi}\left(\cdot, N^{\prime} \Delta t\right)-u\left(\cdot, N^{\prime} \Delta t\right)\right)\right|_{\mathbb{H}} \\
\leqslant & \sqrt{C\left(f, u_{0}, v_{0}\right)}\left|\pi_{h}\left(w_{\eta, \chi}\left(\cdot, N^{\prime} \Delta t\right)-u\left(\cdot, N^{\prime} \Delta t\right)\right)\right|_{\mathbb{H}} \\
\leqslant & \sqrt{C\left(f, u_{0}, v_{0}\right)}\left|\left(\pi_{h}-I d\right)\left(w_{\eta, \chi}\left(\cdot, N^{\prime} \Delta t\right)-u\left(\cdot, N^{\prime} \Delta t\right)\right)\right|_{\mathbb{H}} \\
& +\sqrt{C\left(f, u_{0}, v_{0}\right)}\left|w_{\eta, \chi}\left(\cdot, N^{\prime} \Delta t\right)-u\left(\cdot, N^{\prime} \Delta t\right)\right|_{\mathbb{H}} \cdot
\end{aligned}
$$

Let us recall that, by construction, $w(\cdot, t)=u(\cdot, t)$ for all $t \in[T-3 \varepsilon / 2, T]$ and that $N^{\prime}$ is the integer part of $\frac{T-\varepsilon}{\Delta t}$. So, for $\Delta t$ small enough, it is possible to have $N^{\prime} \Delta t \geqslant$ $T-3 \varepsilon / 2$. Consequently, the definition of $w_{\eta, \chi},(4.5)$, leads to

$$
\begin{aligned}
w_{\eta, \chi}\left(\cdot, N^{\prime} \Delta t\right)-u\left(\cdot, N^{\prime} \Delta t\right) & =\frac{1}{\eta} \int_{N^{\prime} \Delta t}^{N^{\prime} \Delta t+\eta}((1-\chi) w(\cdot, t)-u(\cdot, t)) \mathrm{d} t \\
& =\frac{\chi}{\eta} \int_{N^{\prime} \Delta t}^{N^{\prime} \Delta t+\eta} u(\cdot, t) \mathrm{d} t
\end{aligned}
$$


Moreover, following Step 2 (2), if $\varphi$ belongs to $L^{\infty}(0, T ; \mathbb{H}), a$ and $b$ being such that $0 \leqslant a<b \leqslant T$, one has

$$
\begin{aligned}
\left|\int_{a}^{b} \varphi(\cdot, t) \mathrm{d} t\right|_{\mathbb{H}}^{2} & \leqslant(b-a) \int_{\Omega} \int_{a}^{b} \varphi^{2}(x, t) \mathrm{d} t \mathrm{~d} x \\
& \leqslant(b-a)^{2} \sup _{t}|\varphi(\cdot, t)|_{\mathbb{H}}^{2}=(b-a)^{2}\|\varphi\|_{L^{\infty}(0, T ; \mathbb{H})}^{2},
\end{aligned}
$$

or else

$$
\left|\int_{a}^{b} \varphi(\cdot, t) \mathrm{d} t\right|_{\mathbb{H}} \leqslant(b-a)\|\varphi\|_{L^{\infty}(0, T ; \mathbb{H})} .
$$

As $u$ belongs to $L^{\infty}(0, T ; \mathbb{V})$, this result implies that

$$
\begin{aligned}
\left|w_{\eta, \chi}\left(\cdot, N^{\prime} \Delta t\right)-u\left(\cdot, N^{\prime} \Delta t\right)\right|_{\mathbb{H}} & =\frac{\chi}{\eta}\left|\int_{N^{\prime} \Delta t}^{N^{\prime} \Delta t+\eta} u(\cdot, t) \mathrm{d} t\right|_{\mathbb{H}} \leqslant \chi\|u\|_{L^{\infty}(0, T ; \mathbb{H})} \\
& \leqslant \chi\|u\|_{L^{\infty}(0, T ; \mathbb{V})} .
\end{aligned}
$$

Finally, using (4.7), as $\gamma_{h}$ goes to zero when $h$ goes to zero, we have

$$
\begin{aligned}
\mid\left(\pi_{h}\right. & -I d)\left.\left(w_{\eta, \chi}\left(\cdot, N^{\prime} \Delta t\right)-u\left(\cdot, N^{\prime} \Delta t\right)\right)\right|_{\mathbb{H}} \\
& \leqslant\left\|\left(\pi_{h}-I d\right)\left(w_{\eta, \chi}\left(\cdot, N^{\prime} \Delta t\right)-u\left(\cdot, N^{\prime} \Delta t\right)\right)\right\|_{H^{1+\xi}(\Omega)} \\
& \leqslant\left\|\left(\pi_{h}-I d\right)\left(w_{\eta, \chi}\left(\cdot, N^{\prime} \Delta t\right)-u\left(\cdot, N^{\prime} \Delta t\right)\right)\right\|_{\mathbb{V}} \\
& \leqslant \gamma_{h}\left\|w_{\eta, \chi}-u\right\|_{L^{\infty}(0, T-\varepsilon / 2 ; \mathbb{V})} \leqslant \chi\|u\|_{L^{\infty}(0, T ; \mathbb{V})}
\end{aligned}
$$

if $h$ is chosen small enough. Hence, it leads to

$$
\left|S_{1}\right| \leqslant 2 \chi \sqrt{C\left(f, u_{0}, v_{0}\right)}\|u\|_{L^{\infty}(0, T ; \mathbb{V})} \equiv \chi C\|u\|_{L^{\infty}(0, T ; \mathbb{V})} .
$$

(2) Let us now derive an estimate for $S_{2}$.

$$
\begin{aligned}
\left|S_{2}\right| & =\left|\sum_{n=1}^{N^{\prime}}\left(\int_{\Omega} \frac{u_{n}^{h}-u_{n-1}^{h}}{\Delta t}\left(\pi_{h}-I d\right) \psi_{\Delta t}(\cdot,(n-1) \Delta t) \mathrm{d} x\right) \Delta t\right| \\
& \leqslant \sum_{n=1}^{N^{\prime}}\left|\frac{u_{n}^{h}-u_{n-1}^{h}}{\Delta t}\right|_{\mathbb{H}}\left|\left(\pi_{h}-I d\right) \psi_{\Delta t}(\cdot,(n-1) \Delta t)\right|_{\mathbb{H}} \Delta t \\
& \leqslant \sqrt{C\left(f, u_{0}, v_{0}\right)} \sum_{n=1}^{N^{\prime}}\left|\left(\pi_{h}-I d\right) \psi_{\Delta t}(\cdot,(n-1) \Delta t)\right|_{\mathbb{H}} \Delta t \\
& \left.\leqslant \gamma_{h} \sqrt{C\left(f, u_{0}, v_{0}\right)} \sum_{n=1}^{N^{\prime}}\left\|\psi_{\Delta t}(\cdot,(n-1) \Delta t)\right\|_{\mathbb{V} \Delta t} \Delta\right\rangle_{h} \sqrt{C\left(f, u_{0}, v_{0}\right)} \sqrt{N}\left(\sum_{n=1}^{N^{\prime}}\left\|\Delta t \psi_{\Delta t}(\cdot,(n-1) \Delta t)\right\|_{\mathbb{V}}^{2}\right)^{1 / 2},
\end{aligned}
$$


thanks to (4.2) and (4.7). Moreover, the definitions of $\psi_{\Delta t}$ and $w_{\eta, \chi},(4.5)$, lead to

$$
\begin{aligned}
\left\|\Delta t \psi_{\Delta t}(\cdot,(n-1) \Delta t)\right\|_{\mathbb{V}}^{2}= & \left\|\left(w_{\eta, \chi}-u\right)(x, n \Delta t)-\left(w_{\eta, \chi}-u\right)(x,(n-1) \Delta t)\right\|_{\mathbb{V}}^{2} \\
= & \| \int_{n \Delta t}^{n \Delta t+\eta} \frac{(1-\chi) w(x, t)-u(x, t)}{\eta} \mathrm{d} t \\
& -\int_{(n-1) \Delta t}^{(n-1) \Delta t+\eta} \frac{(1-\chi) w(x, t)-u(x, t)}{\eta} \mathrm{d} t \|_{\mathbb{V}}^{2} \\
= & \| \int_{(n-1) \Delta t+\eta}^{n \Delta t+\eta} \frac{(1-\chi) w(x, t)-u(x, t)}{\eta} \mathrm{d} t \\
& -\int_{(n-1) \Delta t}^{n \Delta t} \frac{(1-\chi) w(x, t)-u(x, t)}{\eta} \mathrm{d} t \|_{\mathbb{V}}^{2} \\
\leqslant & 2\left\|\int_{(n-1) \Delta t+\eta}^{n \Delta t+\eta} \frac{(1-\chi) w(x, t)-u(x, t)}{\eta} \mathrm{d} t\right\|_{\mathbb{V}}^{2} \\
& +2\left\|\int_{(n-1) \Delta t}^{n \Delta t} \frac{(1-\chi) w(x, t)-u(x, t)}{\eta} \mathrm{d} t\right\|_{\mathbb{V}}^{2} . \quad(4.1
\end{aligned}
$$

Now, if $\varphi$ belongs to $L^{2}(0, T ; \mathbb{H})$, one has

$$
\begin{aligned}
\sum_{n=1}^{N^{\prime}}\left|\int_{(n-1) \Delta t}^{n \Delta t} \varphi(\cdot, t) \mathrm{d} t\right|_{\mathbb{H}}^{2} & =\sum_{n=1}^{N^{\prime}} \int_{\Omega}\left(\int_{(n-1) \Delta t}^{n \Delta t} \varphi(x, t) \mathrm{d} t\right)^{2} \mathrm{~d} x \\
& \leqslant \sum_{n=1}^{N^{\prime}} \Delta t \int_{\Omega} \int_{(n-1) \Delta t}^{n \Delta t} \varphi^{2}(x, t) \mathrm{d} t \mathrm{~d} x \\
& \leqslant \Delta t \int_{\Omega} \int_{0}^{T} \varphi^{2}(x, t) \mathrm{d} t \mathrm{~d} x=\Delta t\|\varphi\|_{L^{2}(0, T ; \mathbb{H})}^{2}
\end{aligned}
$$

In a similar way, as $\eta<\varepsilon / 2$ and $N^{\prime} \Delta t \leqslant T-\varepsilon$ (from the definition of $N^{\prime}$ ), we have $N^{\prime} \Delta t+\eta \leqslant T$ and then

$$
\sum_{n=1}^{N^{\prime}}\left|\int_{(n-1) \Delta t+\eta}^{n \Delta t+\eta} \varphi(\cdot, t) \mathrm{d} t\right|_{\mathbb{H}}^{2} \leqslant \Delta t\|\varphi\|_{L^{2}(0, T ; \mathbb{H})}^{2} .
$$

If $\varphi$ belongs to $L^{2}(0, T ; \mathbb{V})$, the previous properties can be extended to its space derivatives (in the distribution sense) in exactly the same way, leading to

$$
\left(\sum_{n=1}^{N^{\prime}}\left\|\int_{(n-1) \Delta t}^{n \Delta t} \varphi(\cdot, t) \mathrm{d} t\right\|_{\mathbb{V}}^{2}+\sum_{n=1}^{N^{\prime}}\left\|\int_{(n-1) \Delta t+\eta}^{n \Delta t+\eta} \varphi(\cdot, t) \mathrm{d} t\right\|_{\mathbb{V}}^{2}\right)^{1 / 2} \leqslant \sqrt{2 \Delta t}\|\varphi\|_{L^{2}(0, T ; \mathbb{V})} .
$$


Setting $\varphi=\frac{1}{\eta}((1-\chi) w-u)$ in the above inequality, this result and (4.15) imply that

$$
\begin{aligned}
\left|S_{2}\right| & \leqslant \gamma_{h} \sqrt{C\left(f, u_{0}, v_{0}\right)} \sqrt{N}\left(\sum_{n=1}^{N^{\prime}}\left\|\Delta t \psi_{\Delta t}(\cdot,(n-1) \Delta t)\right\|_{\mathbb{V}}^{2}\right)^{1 / 2} \\
& \leqslant \gamma_{h} \sqrt{C\left(f, u_{0}, v_{0}\right)} \sqrt{N} \frac{2 \sqrt{\Delta t}}{\eta}\|(1-\chi) w-u\|_{L^{2}(0, T ; \mathbb{V})} \\
& \leqslant \gamma_{h} \sqrt{C\left(f, u_{0}, v_{0}\right)} \frac{2 \sqrt{T}}{\eta}\|(1-\chi) w-u\|_{L^{2}(0, T ; \mathbb{V})} \stackrel{h \rightarrow 0}{\longrightarrow} 0 .
\end{aligned}
$$

(3) To treat the third term, we begin with the following transformation. First, let us recall that the definitions of $\psi_{\Delta t}$ and $w_{\eta, \chi}$ lead, for all $\tau \in[0, T-\varepsilon / 2]$, to

$$
\begin{aligned}
\psi_{\Delta t}(x, \tau)= & \frac{\left(w_{\eta, \chi}-u\right)(x, \tau+\Delta t)-\left(w_{\eta, \chi}-u\right)(x, \tau)}{\Delta t} \\
= & \frac{1}{\eta \Delta t} \int_{\tau+\Delta t}^{\tau+\Delta t+\eta}((1-\chi) w-u)(x, s) \mathrm{d} s \\
& -\frac{1}{\eta \Delta t} \int_{\tau}^{\tau+\eta}((1-\chi) w-u)(x, s) \mathrm{d} s \\
= & \frac{1}{\eta \Delta t} \int_{\tau}^{\tau+\eta}(((1-\chi) w-u)(x, s+\Delta t)-((1-\chi) w-u)(x, s)) \mathrm{d} s \\
= & \frac{1}{\eta \Delta t} \int_{\tau}^{\tau+\eta}\left(\int_{s}^{s+\Delta t}((1-\chi) \dot{w}-\dot{u})(x, r) \mathrm{d} r\right) \mathrm{d} s .
\end{aligned}
$$

Hence, we obtain

$$
\begin{array}{r}
\psi_{\Delta t}(x,(n-1) \Delta t)-\psi_{\Delta t}(x, t) \\
=\frac{1}{\eta \Delta t}\left(\int_{(n-1) \Delta t}^{(n-1) \Delta t+\eta} \int_{s}^{s+\Delta t}((1-\chi) \dot{w}-\dot{u})(x, r) \mathrm{d} r \mathrm{~d} s\right. \\
\left.\quad-\int_{t}^{t+\eta} \int_{s}^{s+\Delta t}((1-\chi) \dot{w}-\dot{u})(x, r) \mathrm{d} r \mathrm{~d} s\right) \\
=\frac{1}{\eta \Delta t}\left(\int_{(n-1) \Delta t}^{t} \int_{s}^{s+\Delta t}((1-\chi) \dot{w}-\dot{u})(x, r) \mathrm{d} r \mathrm{~d} s\right. \\
\left.\quad-\int_{(n-1) \Delta t+\eta}^{t+\eta} \int_{s}^{s+\Delta t}((1-\chi) \dot{w}-\dot{u})(x, r) \mathrm{d} r \mathrm{~d} s\right) .
\end{array}
$$


Now, $a$ and $b$ being such that $0 \leqslant a<b \leqslant T$, and setting $\varphi=(1-\chi) \dot{w}-\dot{u}$, where $\phi$ belongs to $L^{2}(0, T ; \mathbb{H})$, one has

$$
\begin{aligned}
\left|\int_{a}^{b} \int_{s}^{s+\Delta t} \varphi(\cdot, r) \mathrm{d} r \mathrm{~d} s\right|_{\mathbb{H}}^{2} & =\int_{\Omega}\left(\int_{a}^{b} \int_{s}^{s+\Delta t} \varphi(x, r) \mathrm{d} r \mathrm{~d} s\right)^{2} \mathrm{~d} x \\
& \leqslant \int_{\Omega}\left(\int_{a}^{b} \sqrt{\Delta t}\left[\int_{s}^{s+\Delta t} \varphi^{2}(x, r) \mathrm{d} r\right]^{1 / 2} \mathrm{~d} s\right)^{2} \mathrm{~d} x \\
& \leqslant \int_{\Omega}(b-a) \Delta t \int_{a}^{b} \int_{s}^{s+\Delta t} \varphi^{2}(x, r) \mathrm{d} r \mathrm{~d} s \mathrm{~d} x \\
& =(b-a) \Delta t \int_{a}^{b}\left(\int_{\Omega} \int_{s}^{s+\Delta t} \varphi^{2}(x, r) \mathrm{d} r \mathrm{~d} x\right) \mathrm{d} s \\
& \leqslant(b-a)^{2} \Delta t\|\varphi\|_{L^{2}(0, T ; \mathbb{H})}
\end{aligned}
$$

and then

$$
\left|\psi_{\Delta t}(\cdot,(n-1) \Delta t)-\psi_{\Delta t}(\cdot, t)\right|_{\mathbb{H}} \leqslant 2 \frac{|t-(n-1) \Delta t|}{\eta \sqrt{\Delta t}}\|((1-\chi) \dot{w}-\dot{u})\|_{L^{2}(0, T ; \mathbb{H})} .
$$

Finally, using (4.2) again, we obtain from these results

$$
\begin{aligned}
\left|S_{3}\right| & \leqslant \sum_{n=1}^{N^{\prime}} \int_{(n-1) \Delta t}^{n \Delta t}\left|\frac{u_{n}^{h}-u_{n-1}^{h}}{\Delta t}\right|_{\mathbb{H}}\left|\psi_{\Delta t}(\cdot,(n-1) \Delta t)-\psi_{\Delta t}(\cdot, t)\right|_{\mathbb{H}} \mathrm{d} t \\
& \leqslant \sqrt{C\left(f, u_{0}, v_{0}\right)} \sum_{n=1}^{N^{\prime}} \int_{(n-1) \Delta t}^{n \Delta t} 2 \frac{|t-(n-1) \Delta t|}{\eta \sqrt{\Delta t}}\|(1-\chi) \dot{w}-\dot{u}\|_{L^{2}(0, T ; \mathbb{H})} \mathrm{d} t \\
& \leqslant \sqrt{C\left(f, u_{0}, v_{0}\right)} \sum_{n=1}^{N^{\prime}} \frac{(\Delta t)^{2}}{\eta \sqrt{\Delta t}}\|(1-\chi) \dot{w}-\dot{u}\|_{L^{2}(0, T ; \mathbb{H})} \\
& \leqslant \sqrt{C\left(f, u_{0}, v_{0}\right)} \frac{T \sqrt{\Delta t}}{\eta}\|(1-\chi) \dot{w}-\dot{u}\|_{L^{2}(0, T ; \mathbb{H})} \stackrel{\Delta t \rightarrow 0}{\rightarrow} 0 .
\end{aligned}
$$

(4) Finally, from the definition of $u_{h, N},(4.3)$, we have $\dot{u}_{h, N}(x, t)=\frac{u_{n}^{h}(x)-u_{n-1}^{h}(x)}{\Delta t}$ where $t$ belongs to $[(n-1) \Delta t, n \Delta t]$. Hence, $S_{4}$ can be rewritten

$$
\begin{aligned}
S_{4} & =\sum_{n=1}^{N^{\prime}} \int_{(n-1) \Delta t}^{n \Delta t} \int_{\Omega} \frac{u_{n}^{h}-u_{n-1}^{h}}{\Delta t} \psi_{\Delta t}(\cdot, t) \mathrm{d} x \mathrm{~d} t \\
& =\int_{0}^{T-\varepsilon} \int_{\Omega} \dot{u}_{h, N} \psi_{\Delta t} \mathrm{~d} x \mathrm{~d} t-\int_{N^{\prime} \Delta t}^{T-\varepsilon} \int_{\Omega} \frac{u_{N^{\prime}}^{h}-u_{N^{\prime}-1}^{h}}{\Delta t} \psi_{\Delta t}(\cdot, t) \mathrm{d} x \mathrm{~d} t .
\end{aligned}
$$


Exactly as for the previous point, using (4.2) and (4.16)-(4.17), we obtain

$$
\begin{aligned}
& \left|\int_{N^{\prime} \Delta t}^{T-\varepsilon} \int_{\Omega} \frac{u_{N^{\prime}}^{h}-u_{N^{\prime}-1}^{h}}{\Delta t} \psi_{\Delta t}(\cdot, t) \mathrm{d} x \mathrm{~d} t\right| \\
& \quad \leqslant \int_{N^{\prime} \Delta t}^{T-\varepsilon}\left|\frac{u_{N^{\prime}}^{h}-u_{N^{\prime}-1}^{h}}{\Delta t}\right|_{\mathbb{H}}|\psi \Delta t(\cdot, t)|_{\mathbb{H}} \mathrm{d} t \\
& \quad \leqslant \int_{N^{\prime} \Delta t}^{T-\varepsilon} \sqrt{C\left(f, u_{0}, v_{0}\right)} \frac{1}{\sqrt{\Delta t}}\|(1-\chi) \dot{w}-\dot{u}\|_{L^{2}(0, T ; \mathbb{H})} \mathrm{d} t \\
& \quad \leqslant \sqrt{C\left(f, u_{0}, v_{0}\right)} \sqrt{\Delta t}\|(1-\chi) \dot{w}-\dot{u}\|_{L^{2}(0, T ; \mathbb{H})} \stackrel{\Delta t \rightarrow{ }^{0} 0 .}{ } .
\end{aligned}
$$

Moreover, following (4.16) with $\tau=t$ belonging to $[0, T-\varepsilon]$, we have

$$
\begin{aligned}
\psi_{\Delta t}(x, t) & =\frac{1}{\eta \Delta t} \int_{t+\Delta t}^{t+\Delta t+\eta}((1-\chi) w-u)(x, s) \mathrm{d} s-\frac{1}{\eta \Delta t} \int_{t}^{t+\eta}((1-\chi) w-u)(x, s) \mathrm{d} s \\
& =\frac{1}{\eta \Delta t} \int_{t+\eta}^{t+\eta+\Delta t}((1-\chi) w-u)(x, s) \mathrm{d} s-\frac{1}{\eta \Delta t} \int_{t}^{t+\Delta t}((1-\chi) w-u)(x, s) \mathrm{d} s \\
& \stackrel{\rightarrow t}{\longrightarrow} \frac{1}{\eta}(((1-\chi) w-u)(x, t+\eta)-((1-\chi) w-u)(x, t)),
\end{aligned}
$$

and this convergence is strong in $L^{2}(0, T-\varepsilon ; \mathbb{V})$ as $(1-\chi) w-u \in L^{2}(0, T ; \mathbb{V})$. Furthermore, as the inclusion of $L^{\infty}(0, T ; \mathbb{H})$ into $L^{2}(0, T ; \mathbb{H})$ is continuous, functions $\left(\dot{u}_{h, N}\right)_{h>0, N \geqslant 1}$ being uniformly bounded in $L^{\infty}(0, T ; \mathbb{H})$ are also uniformly bounded in $L^{2}(0, T ; \mathbb{H})$. So, up to a possible subsequence extraction, $\left(\dot{u}_{h, N}\right)_{h>0, N \geqslant 1}$ converges weakly in this space towards $\dot{u}$ (uniqueness of the limit). So that we obtain

$$
\begin{aligned}
\int_{0}^{T-\varepsilon} & \int_{\Omega} \dot{u}_{h, N} \psi \Delta t \mathrm{~d} x \mathrm{~d} t \\
& \stackrel{h, \Delta t \rightarrow 0}{\longrightarrow} \int_{0}^{T-\varepsilon} \int_{\Omega} \dot{u}(x, t) \frac{((1-\chi) w-u)(x, t+\eta)-((1-\chi) w-u)(x, t)}{\eta} \mathrm{d} x \mathrm{~d} t,
\end{aligned}
$$

and then

$$
S_{2}+S_{3}+S_{4} \stackrel{h, \Delta t \rightarrow}{\longrightarrow} \int_{0}^{T-\varepsilon} \int_{\Omega} \dot{u}(x, t) \frac{((1-\chi) w-u)(x, t+\eta)-((1-\chi) w-u)(x, t)}{\eta} \mathrm{d} x \mathrm{~d} t .
$$

\subsection{Conclusion}

Thanks to the previous convergence results (4.10), (4.11), (4.13), (4.14) and (4.18), when $h$ and $\Delta t$ tend to zero in inequality (4.9), we obtain for all $\varepsilon \in] 0, T / 2[$ and $\eta \in] 0, \varepsilon / 2[$,

$$
\begin{aligned}
& \int_{\Omega} v_{0}(x)\left(w_{\eta, \chi}(x, 0)-u(x, 0)\right) \mathrm{d} x+\int_{0}^{T-\varepsilon} \int_{\Omega} f\left(w_{\eta, \chi}-u\right) \mathrm{d} x \mathrm{~d} t \\
& \leqslant \int_{0}^{T-\varepsilon} a\left(u(\cdot, t),\left(w_{\eta, \chi}-u\right)(\cdot, t)\right) \mathrm{d} t+\chi C\|u\|_{L^{\infty}(0, T ; \mathbb{V})} \\
& \quad-\int_{0}^{T-\varepsilon} \int_{\Omega} \dot{u}(x, t) \frac{((1-\chi) w-u)(x, t+\eta)-((1-\chi) w-u)(x, t)}{\eta} \mathrm{d} x \mathrm{~d} t .
\end{aligned}
$$




$$
\begin{aligned}
& \int_{t}^{t+\eta} \frac{(1-\chi) \dot{w}(x, s)-\dot{u}(x, s)}{\eta} \mathrm{d} s \\
& \stackrel{\eta \rightarrow 0}{\longrightarrow}(1-\chi) \dot{w}(x, t)-\dot{u}(x, t) \quad \text { strongly in } L^{2}(0, T-\varepsilon ; \mathbb{H}) .
\end{aligned}
$$

With the same arguments, as $(1-\chi) w-u \in L^{2}(0, T ; \mathbb{V}) \cap \mathcal{C}^{0}(0, T ; \mathbb{H})$ first we have

$$
\begin{aligned}
w_{\eta, \chi}(x, t)-u(x, t) & =\frac{1}{\eta} \int_{t}^{t+\eta}((1-\chi) w(x, s)-u(x, s)) \mathrm{d} s \\
& \stackrel{\eta \rightarrow 0}{\longrightarrow}(1-\chi) w(x, t)-u(x, t) \quad \text { strongly in } L^{2}(0, T-\varepsilon ; \mathbb{V}),
\end{aligned}
$$

and second, for $t=0$,

$$
w_{\eta, \chi}(x, 0)-u(x, 0) \stackrel{\eta \rightarrow 0}{\longrightarrow}(1-\chi) w(x, 0)-u(x, 0) \text { strongly in } \mathbb{H} .
$$

So, when $\eta$ goes to zero, inequality (4.19) becomes

$$
\begin{aligned}
& \int_{\Omega} v_{0}(x)((1-\chi) w(x, 0)-u(x, 0)) \mathrm{d} x+\int_{0}^{T-\varepsilon} \int_{\Omega} f((1-\chi) w-u) \mathrm{d} x \mathrm{~d} t \\
& \leqslant \int_{0}^{T-\varepsilon} a(u(\cdot, t),((1-\chi) w-u)(\cdot, t)) \mathrm{d} t+\chi C\|u\|_{L^{\infty}(0, T ; \mathbb{V})} \\
& \quad-\int_{0}^{T-\varepsilon} \int_{\Omega} \dot{u}((1-\chi) \dot{w}-\dot{u}) \mathrm{d} x \mathrm{~d} t .
\end{aligned}
$$

The proof is achieved by making $\chi$ and $\varepsilon$ tend to zero, observing that $w-u=\phi(\tilde{w}-u)$, where $\phi$ is defined by (4.4).

REMARK 4.4 Let us recall that, in their paper Dumont \& Paoli (2006), Dumont and Paoli gave a more general result, including, in particular, a conditional convergence when the parameter $\beta$ belongs to $[0,1 / 2[$. Actually, we could have followed the same way. As a matter of fact, the coefficient

$$
\kappa_{h}=\sup _{u^{h} \in \mathbb{V}^{h} \backslash\{0\}} \frac{a\left(u^{h}, u^{h}\right)}{\left|u^{h}\right|_{\mathbb{H}}^{2}}
$$

that they introduced in Dumont \& Paoli (2006) to lead to a conditional stability, can be used in a similar way for plates. It means that Lemma 4.1, which states that the discrete solution is uniformly bounded in time, can also be straightforwardly obtained from Dumont \& Paoli (2006, Proposition 3.1) under the same hypotheses. Then, up to some technical details, if we follow Dumont and Paoli's proof more closely, Theorem 4.2 remains valid. The only point to discuss is the evaluation of $\kappa_{h}$. In Dumont \& Paoli (2006), the authors show that $\kappa_{h}^{\text {beam }} \sim \frac{E I}{\rho S} \frac{1}{\Delta x^{4}}$ for a homogeneous and isotropic beam, $\Delta x$ being the mesh size, which is uniform here. In the case of a Kirchhoff-Love plate, if we assume it is made of a homogeneous and isotropic material too, then the definition of the bilinear form $a(\cdot, \cdot)$ shows that $\kappa_{h}$ is the highest eigenvalue of the bi-Laplacian operator on the plate mesh. So, first, it is proportional to 
$\frac{D}{2 \rho \varepsilon}=\frac{E \varepsilon^{2}}{3\left(1-v^{2}\right) \rho}$. Second, if the mesh is uniform of size $h$, following, for example, Maury (2010), it is easy to see that the highest eigenvalue of the bi-Laplacian is of order $1 / h^{4}$. Consequently, in our case and under the previous assumptions, $\kappa_{h}^{\text {plate }} \sim \frac{E \varepsilon^{2}}{3\left(1-v^{2}\right) \rho} \frac{1}{h^{4}}$, which is quite close to the case of beams. Finally, from a practical point of view, for a similar computational cost, it is better to use an unconditionally stable scheme. Consequently, we only tested the scheme with $\beta=1 / 2$.

\section{Numerical results and conclusions}

We will consider a steel rectangular panel of other lengths all given in metres; change these for consistency. The flexural rigidity is $D=1.923 \times 10^{4}$ corresponding to $E=210 \mathrm{Gpa}, v=0.3$ and $\rho=7.77 \times 10^{3} \mathrm{~kg} / \mathrm{m}^{3}$. This plate is clamped along one edge and free along the other three. The numerical tests are performed with GETFEM++ (Renard \& Pommier, 2003-2012) and using structured meshes (see Figs 3 and 4).

Let us recall the problem to be solved at each iteration:

$$
\left\{\begin{array}{l}
\text { find } u_{n+1}^{h} \in \mathbb{K}^{h} \text { such that } \\
\left(\mathbf{M}+\beta \Delta t^{2} \mathbf{K}\right) u_{n+1}^{h}+\Delta t^{2} \partial \mathbb{I}_{\mathbb{K}^{h}}\left(u_{n+1}^{h}\right) \ni f_{n}^{h} \\
\text { where } f_{n}^{h}=\left(2 \mathbf{M}-(1-2 \beta) \Delta t^{2} \mathbf{K}\right) u_{n}^{h}-\left(\mathbf{M}+\beta \Delta t^{2} \mathbf{K}\right) u_{n-1}^{h}+\Delta t^{2} f^{n \beta}
\end{array}\right.
$$

In practice, we choose $\beta=1 / 2$ in all the following computations. Since the matrix $\mathbf{A} \equiv \mathbf{M}+\beta \Delta t^{2} \mathbf{K}$ is symmetric and positive definite, as are $\mathbf{M}$ and $\mathbf{K}$, this problem is equivalent to the minimization problem

$$
u_{n+1}^{h}=\underset{w \in \mathbb{K}^{h}}{\operatorname{Argmin}}\left(\frac{1}{2} w^{\mathrm{T}} \mathbf{A} w-w^{\mathrm{T}} f_{n}^{h}\right)
$$

As the convex constraints $w \in \mathbb{K}^{h}$ correspond to linear inequality constraints, such a problem can be solved by using the method of Lagrange multipliers or interior point methods, for instance. Here, as

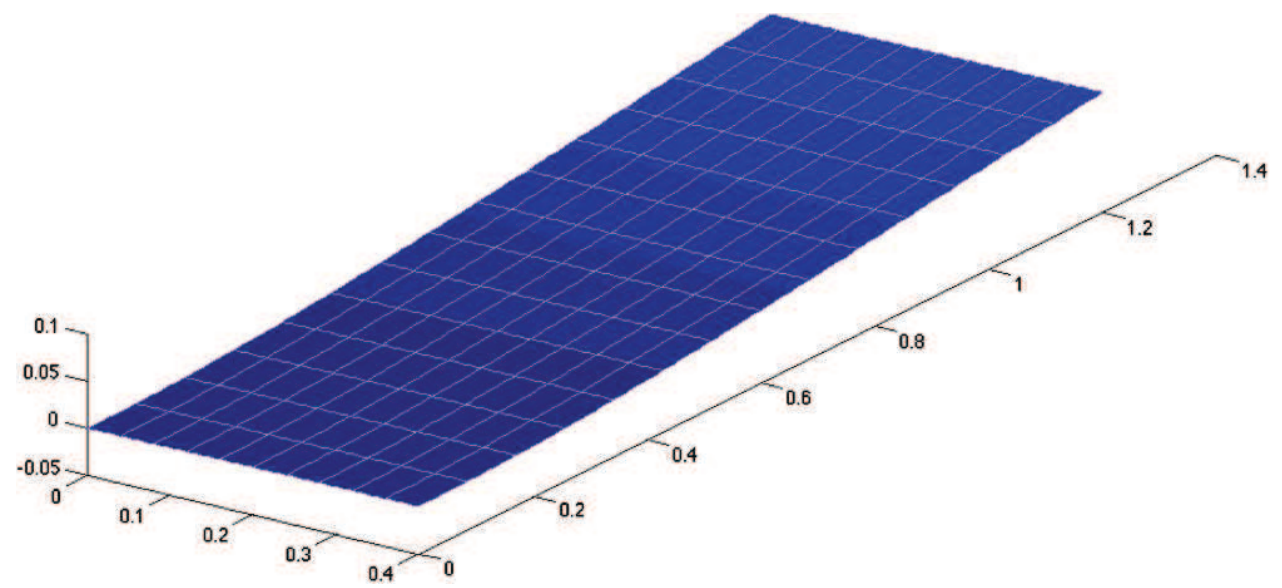

FIG. 3. Bending clamped plate under a rigid obstacle: FVS quadrilateral mesh. 


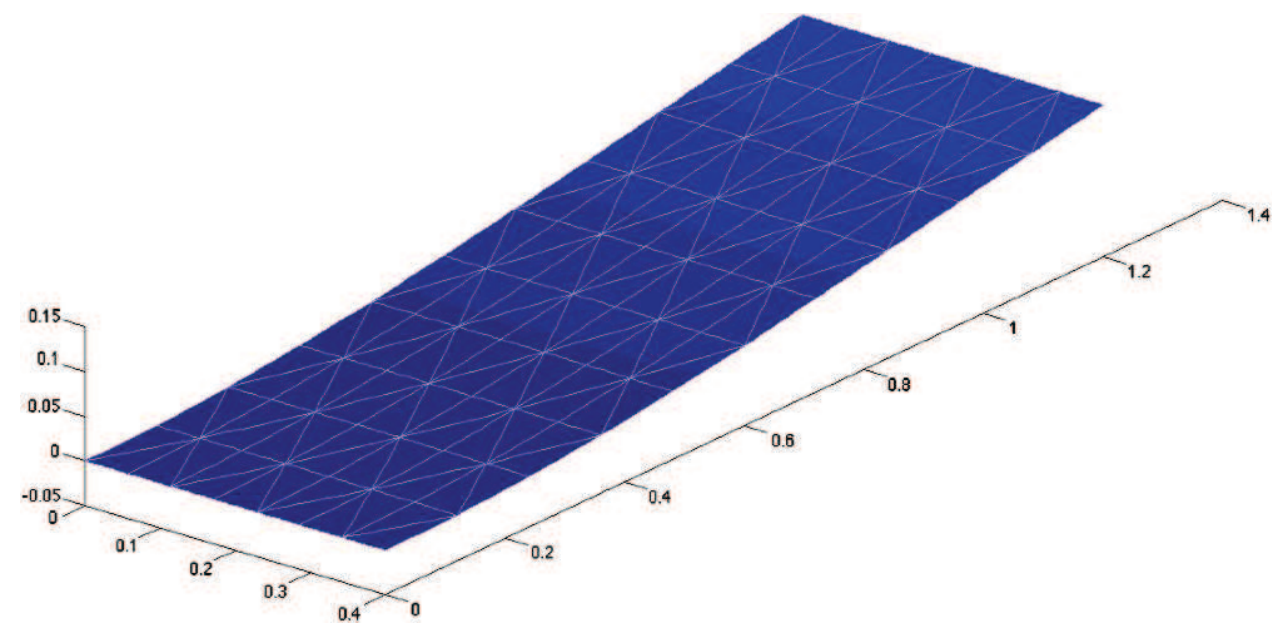

FIG. 4. Bending clamped plate under a rigid obstacle: Argyris triangular mesh.

in Dumont \& Paoli (2008), we use the MATLAB function 'quadprog', which relies on the method of Lagrange multipliers.

\subsection{Forced oscillations}

In this section, we consider two flat, symmetric obstacles along the plate length,

$$
g_{1}(x)=-0.1=-g_{2}(x) \quad \forall x \in \Omega,
$$

and: we prescribe a sine-sweep base forced vibration, by means of the following boundary conditions on $\Gamma_{\mathrm{c}}$

$$
u(x, t)=c \sin (\omega t), \quad \frac{\partial u}{\partial x}(x, t)=0, \quad \forall x \in \Gamma_{\mathrm{c}},
$$

with $c=0.09 \mathrm{~m}$ and $\omega=10 \mathrm{~Hz}$. The displacements of the two free corners, for different time steps and for quadrilateral and triangular meshes, are plotted in Figs 5-7. Not surprisingly, due to the symmetry of the problem, the curves corresponding to the displacements of the two corners, are indistinguishable. Moreover, there is no significant qualitative difference between the FVS and the Argyris approaches. As far as CPU times are concerned, they are given in Table 1 for the numerical simulations related to the previous test case. They are of the same magnitude for triangles and quadrilaterals, considering the fact that the degrees of freedom and the matrix sizes are different. Finally, analogous results to Pozzolini \& Salaun (2011) for a beam impacting obstacles are observed.

To complete this numerical study, some other results are given. First, the case of two flat, symmetric obstacles along the plate where $g_{1}(x)=-0.01=-g_{2}(x)$ for all $x \in \Omega$ is considered in Fig. 8. Second, the case of various frequencies is investigated (see Figs 9-11). All these results confirm the previous conclusions. 


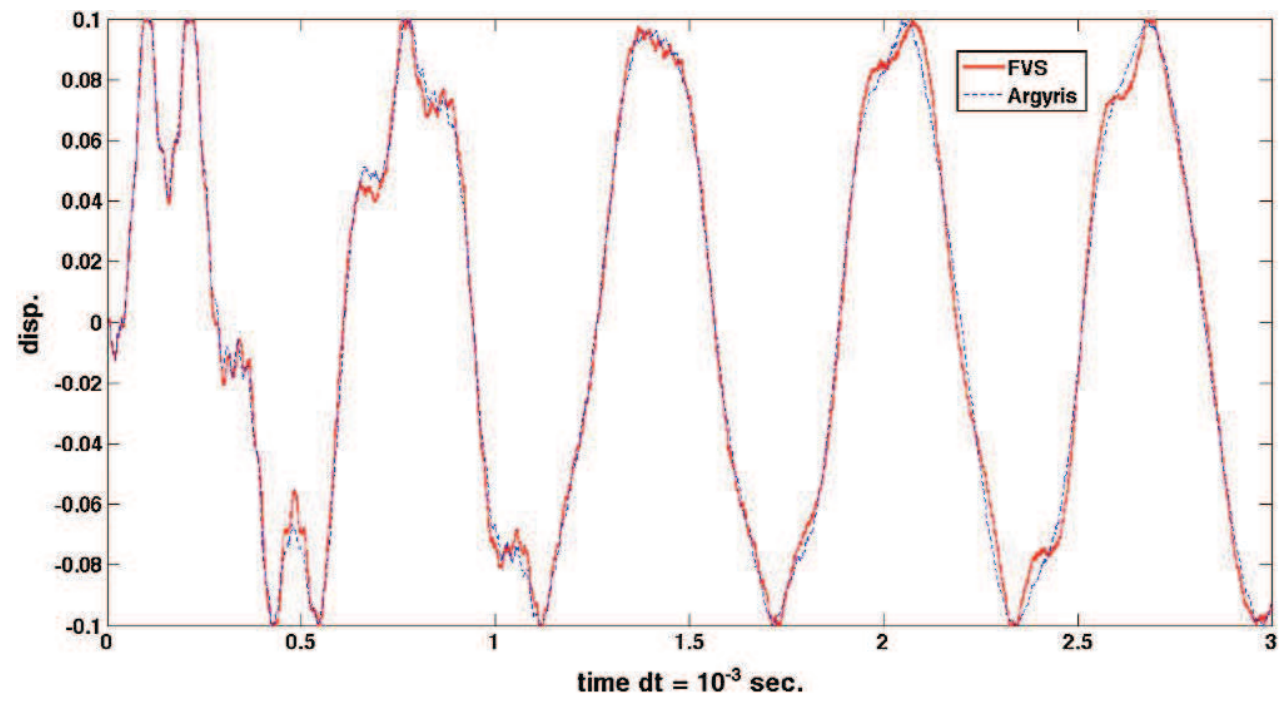

FIG. 5. Displacement of a plate impacting flat obstacles $-140 \mathrm{FVS}$ quadrilaterals and 140 Argyris triangles $-\Delta t=10^{-3}$, obstacle \pm 0.1 .

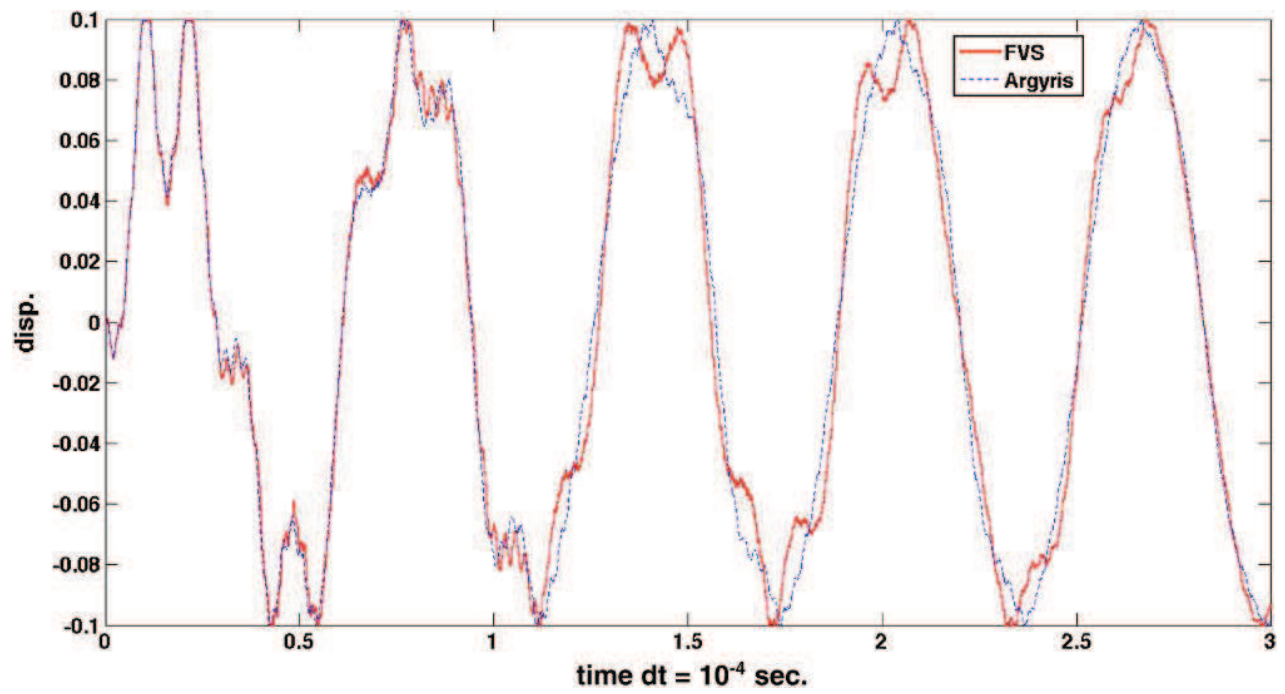

FIG. 6. Displacement of a plate impacting flat obstacles $-140 \mathrm{FVS}$ quadrilaterals and 140 Argyris triangles $-\Delta t=10^{-4}$, obstacle \pm 0.1 .

\subsection{Energy evolution}

This section is devoted to the study of energy variations during the motion. So, here, a forced vibration is not prescribed. The motion is due to an initial displacement $u_{0}$, obtained as the static equilibrium of the plate under a constant load $f_{0}=8600 \mathrm{~N}$ and an initial velocity $v_{0}=0$. Moreover, the upper obstacle is removed, which corresponds to setting $g_{2}=+\infty$. The lower obstacle is flat and remains at $g_{1}=-0.1 \mathrm{~m}$. 


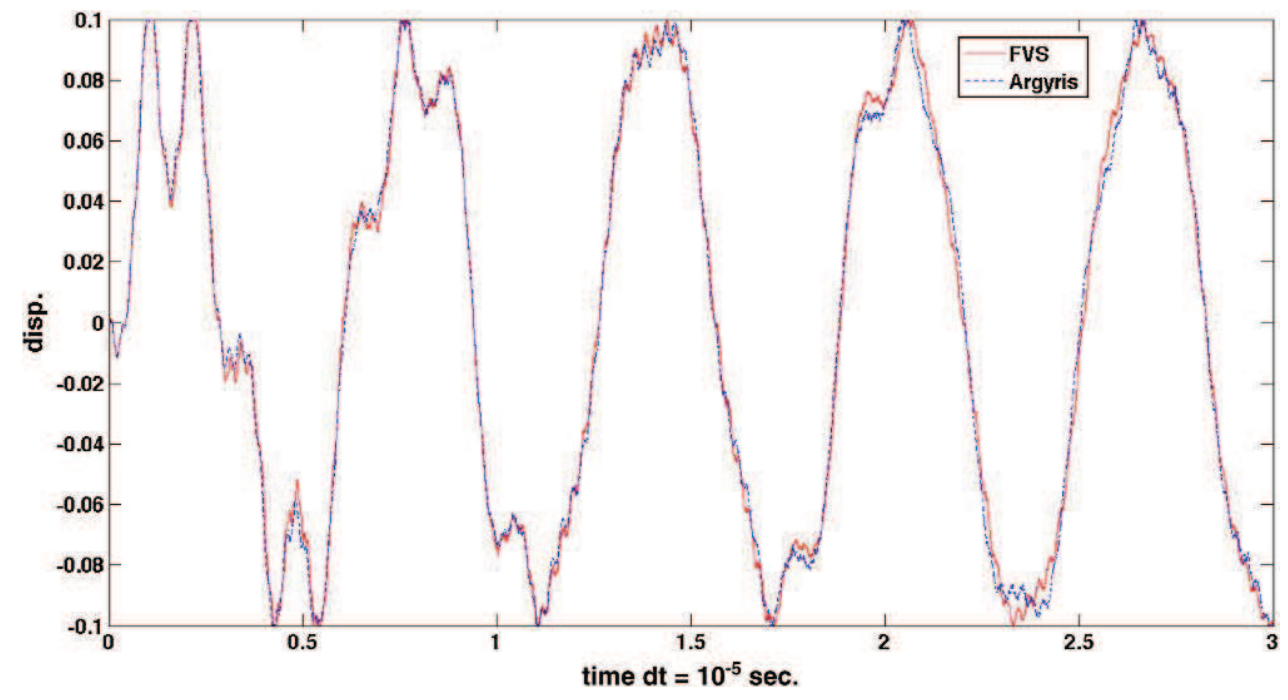

FIG. 7. Displacement of a plate impacting flat obstacles $-140 \mathrm{FVS}$ quadrilaterals and 140 Argyris triangles $-\Delta t=10^{-5}$, obstacle \pm 0.10 .

TABLE 1 CPU times in seconds (MacBook Pro computer with a 2.2 GHz processor)

\begin{tabular}{lrrr}
\hline Time step & $10^{-3}$ & $10^{-4}$ & $10^{-5}$ \\
\hline 140 Argyris triangles & 80 & 870 & 8880 \\
140 FVS quadrilaterals & 120 & 1220 & 12220 \\
\hline
\end{tabular}

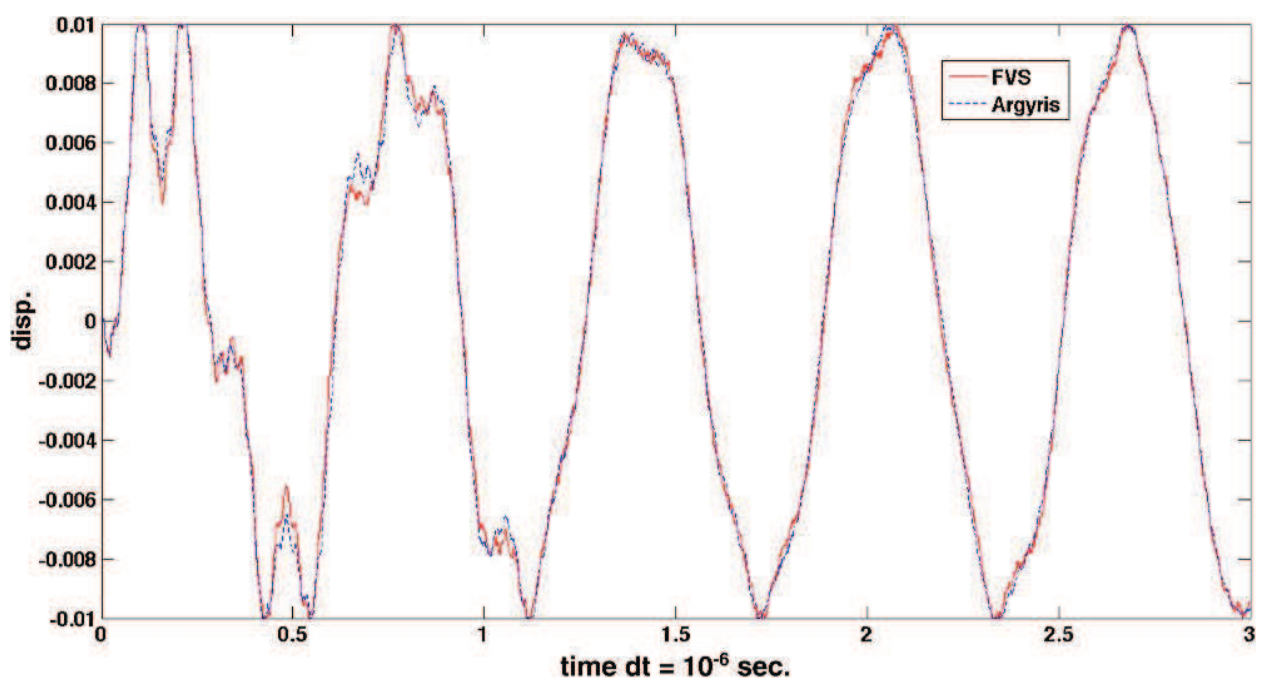

FIG. 8. Displacement of a plate impacting flat obstacles-140 FVS and 140 Argyris elements- $\Delta t=10^{-3}$, obstacle \pm 0.01 . 


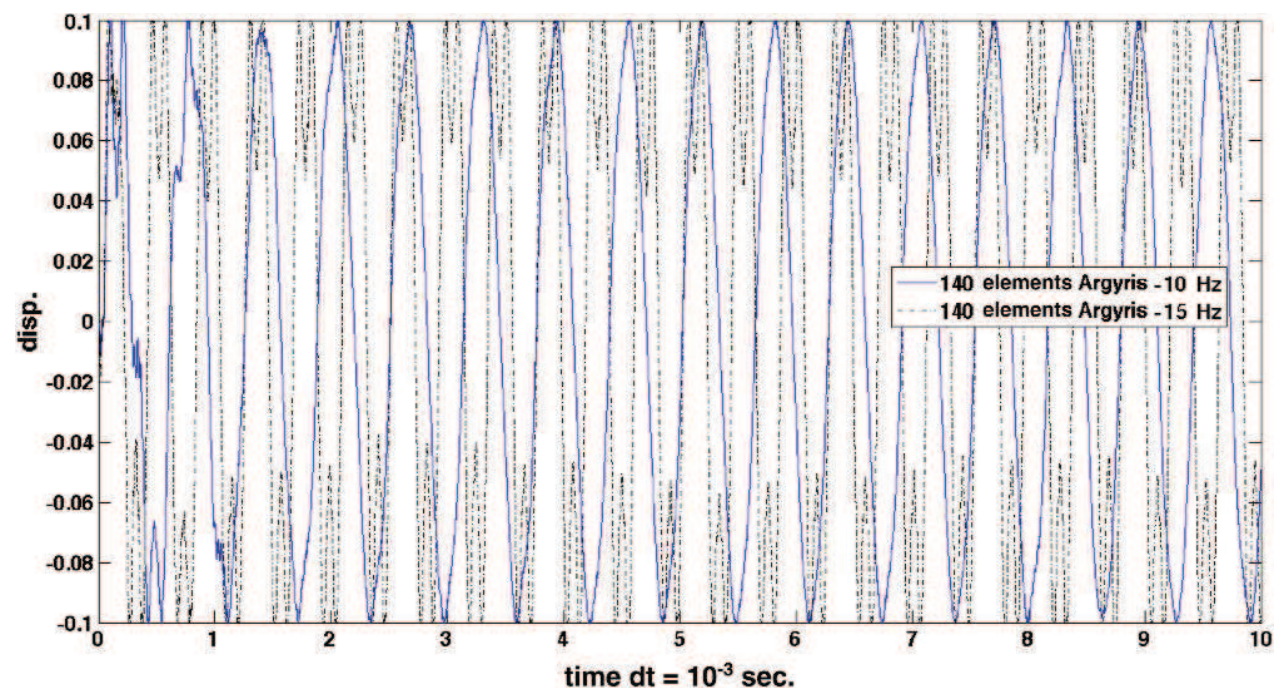

FIG. 9. Displacement of a plate impacting flat obstacles-140 Argyris triangles- $\Delta t=10^{-3}, \omega=10 \mathrm{~Hz}$ and $\omega=15 \mathrm{~Hz}$.

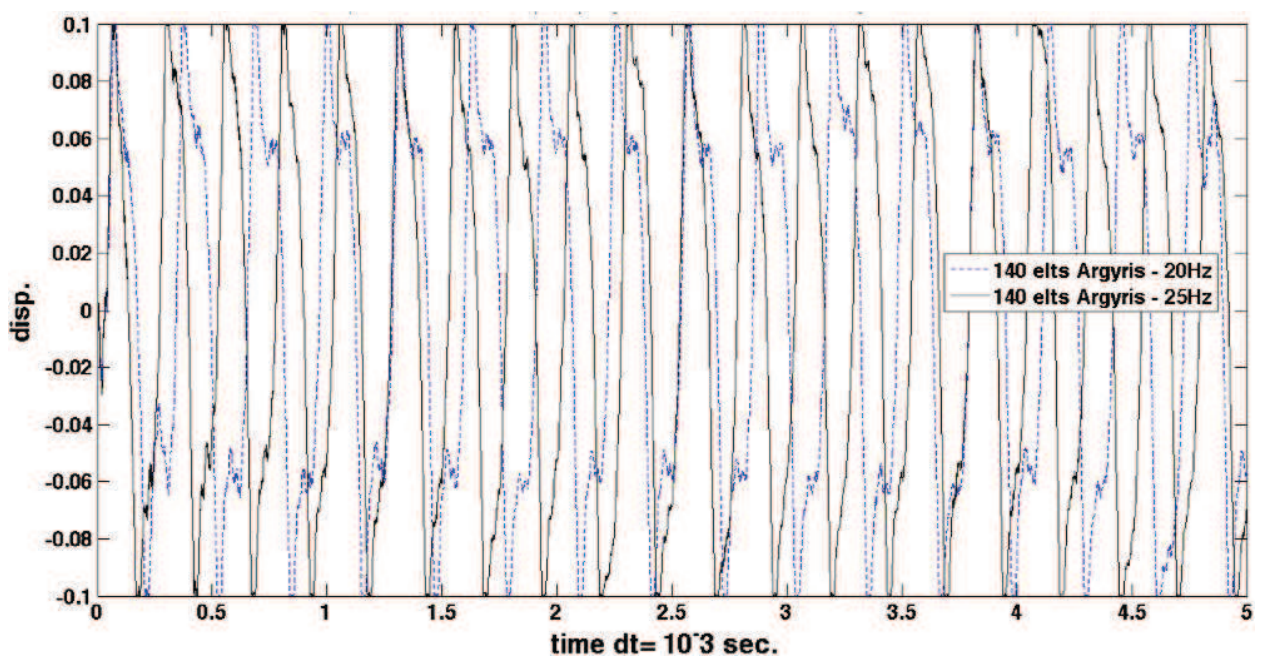

FIG. 10. Displacement of a plate impacting flat obstacles-140 Argyris triangles- $\Delta t=10^{-3}, \omega=20 \mathrm{~Hz}$ and $\omega=25 \mathrm{~Hz}$.

First, as in the previous section, the displacements of the two free corners, and also of their midpoint, are given for meshes of quadrilaterals (Fig. 12) and triangles (Fig. 13). The results are very close. Here again, the three curves are indistinguishable. For the two corners, this was expected, but not for the midpoint. To investigate this, a zoom was created on these curves (Figs 14-16). They show such a small difference in the motion of these three points that this explains why it is not visible in the first figures. Moreover, Figs 15 and 16 illustrate again that there is no meaningful difference between meshes of triangles and quadrilaterals. Finally, Figs 12 and 13 show that the maximum displacements decrease as time passes, which means that impacts create damping during the motion. 


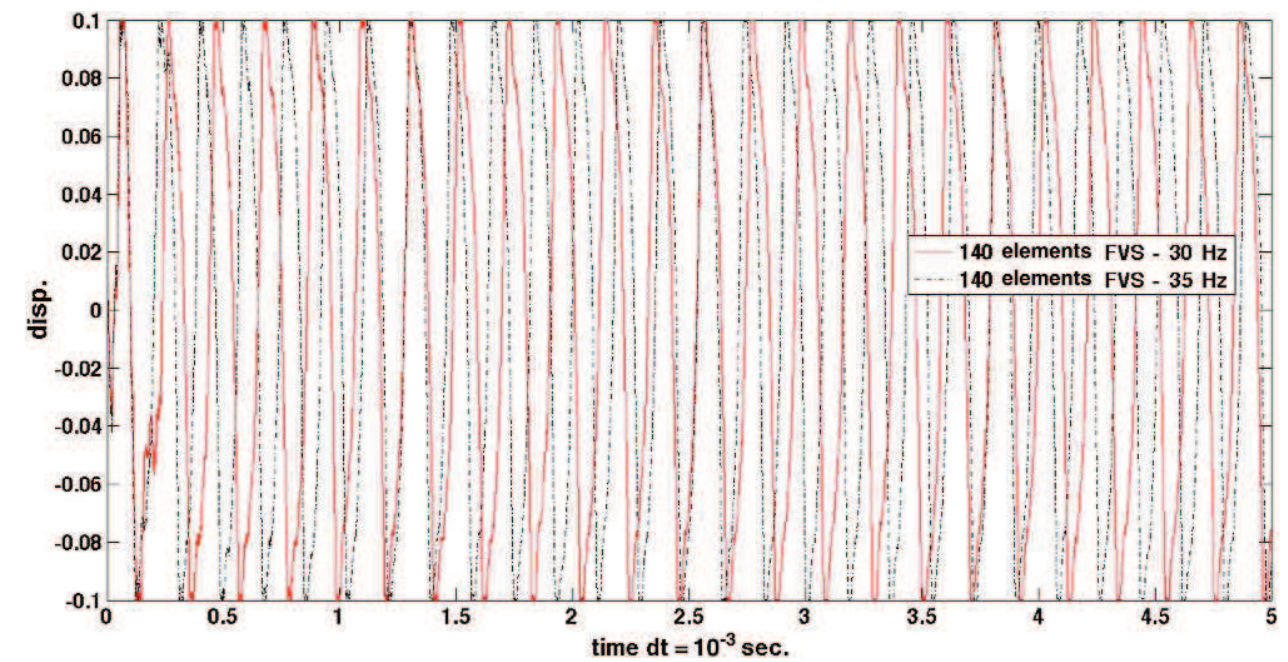

FIG. 11. Displacement of a plate impacting flat obstacles-140 FVS quadrilaterals- $\Delta t=10^{-3}, \omega=30 \mathrm{~Hz}$ and $\omega=35 \mathrm{~Hz}$.

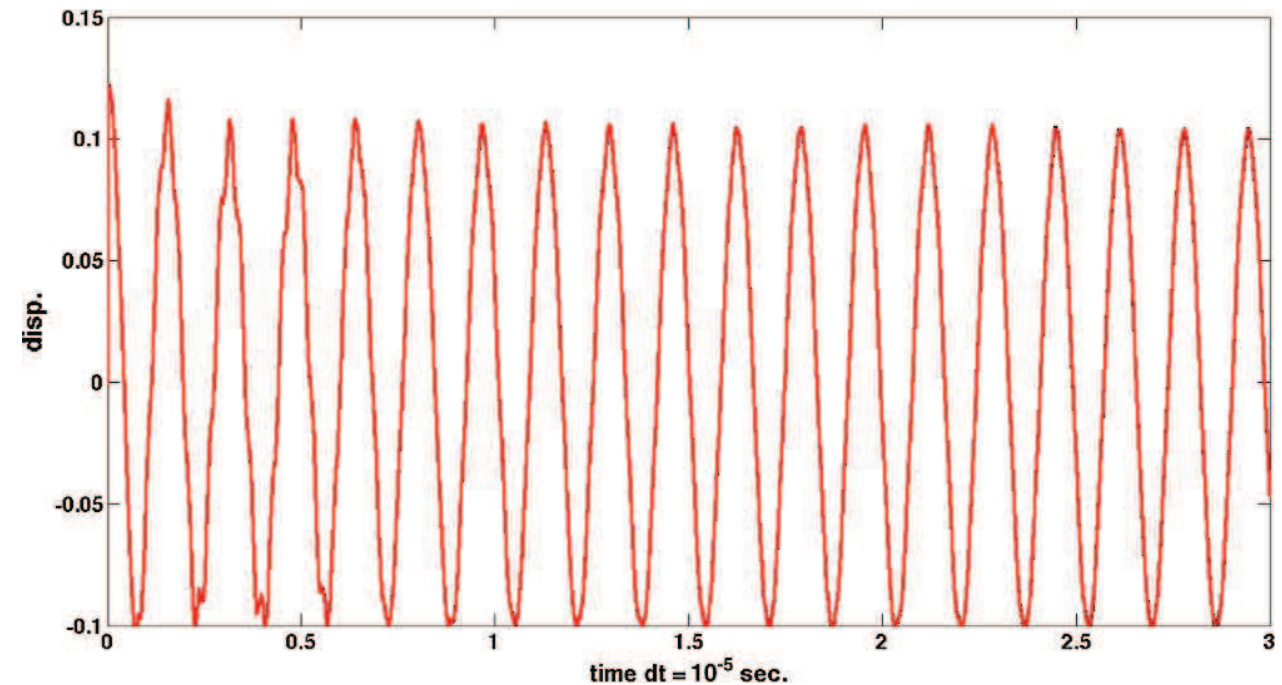

FIG. 12. Displacements in free vibrations $-140 \mathrm{FVS}$ quadrilaterals $-\Delta t=10^{-5}$.

Finally, we compare the variations of the total energy obtained for different time steps and meshes. This total energy is defined by

$$
E(w, t)=\frac{1}{2} \int_{\Omega}(\dot{w})^{2}(x, t) \mathrm{d} x+\frac{1}{2} a(w(\cdot, t), w(\cdot, t))-\int_{\Omega} f(x, t) w(x, t) \mathrm{d} x .
$$

In the case of free vibrations, the loading $f$ is zero. The associated discrete energy is

$$
E\left(u_{n+1}^{h}, u_{n}^{h}\right)=\frac{1}{2} \int_{\Omega}\left|\frac{u_{n+1}^{h}-u_{n}^{h}}{\Delta t}\right|^{2} \mathrm{~d} x+\frac{1}{2} a\left(u_{n}^{h}, u_{n}^{h}\right) .
$$




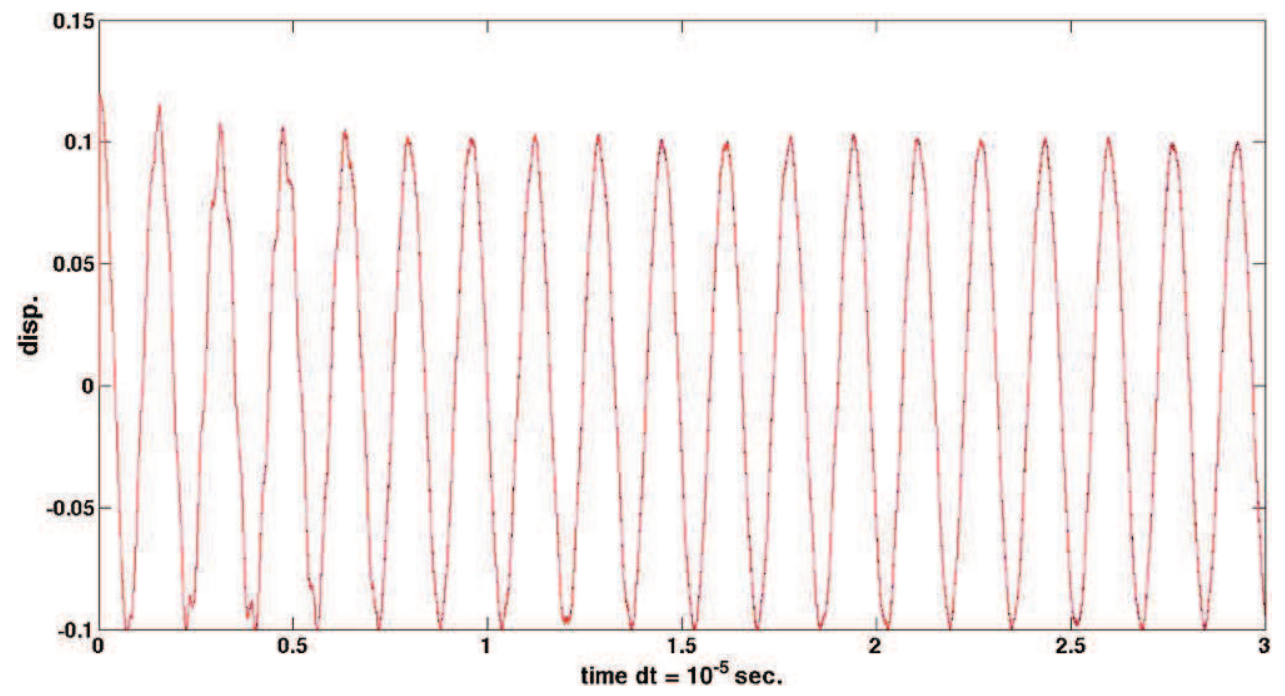

FIG. 13. Displacements in free vibrations-160 Argyris triangles- $\Delta t=10^{-5}$.

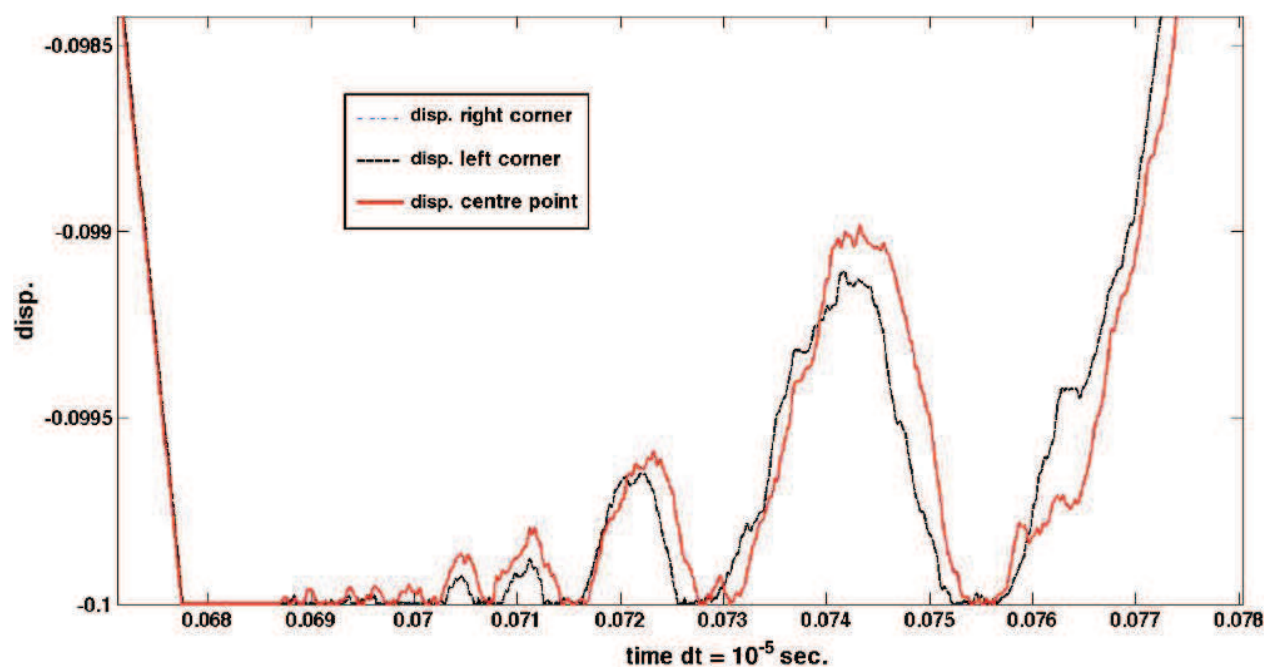

FIG. 14. Zoom on displacements in free vibrations-140 FVS quadrilaterals $-\Delta t=10^{-5}$.

Figures 17 and 18 show the decreases in discrete energy. First of all, let us remark that these curves exhibit a small difference in the initial energy, which is due to difference in discretization between the two meshes. But it is a detail. The main point is that, in the two cases, energy is dissipated when the plate reaches the obstacles. The same qualitative results were obtained in Ahn \& Stewart (2005) and Dumont \& Paoli (2006). By the way, our numerical model is a fully implicit scheme. It seems that it 


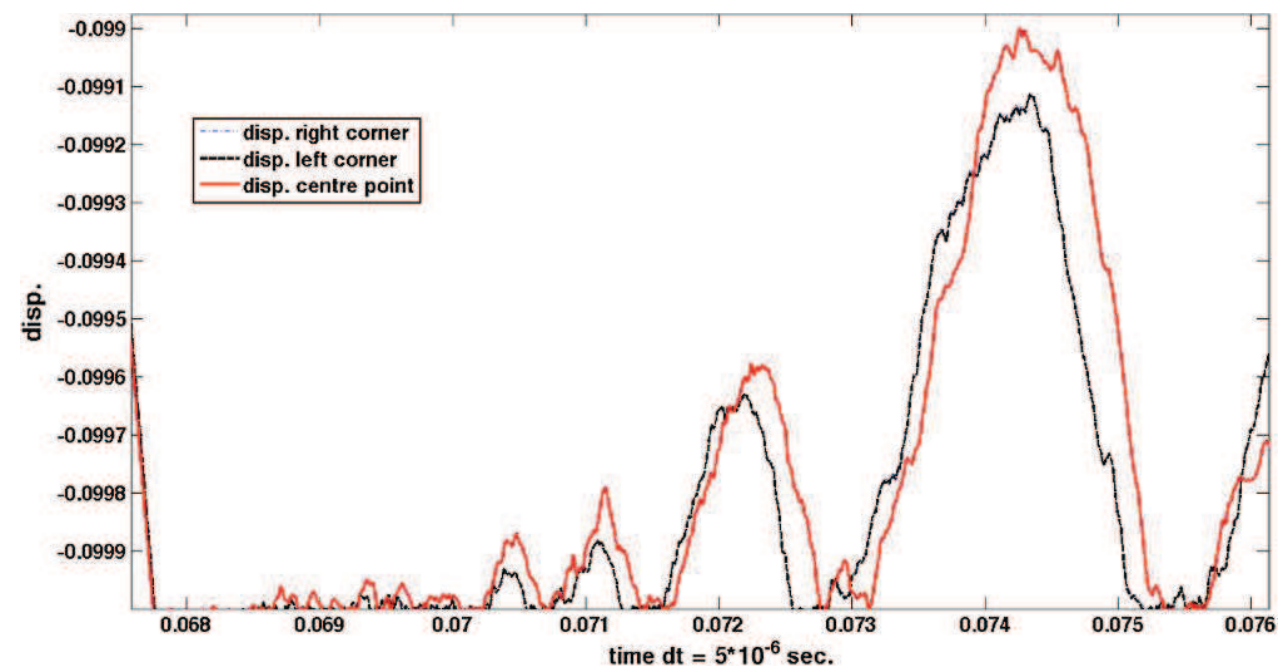

FIG. 15. Zoom on displacements in free vibrations-140 FVS quadrilaterals- $\Delta t=5 \times 10^{-6}$.

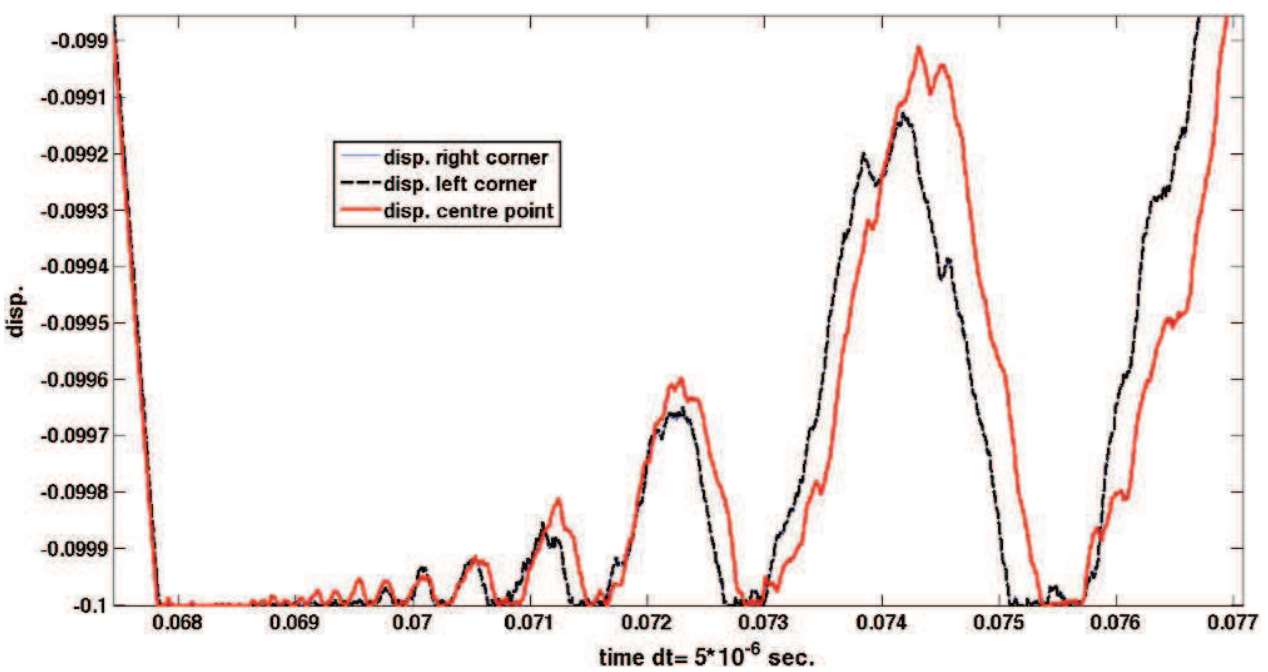

FIG. 16. Zoom on displacements in free vibrations-160 Argyris triangles- $\Delta t=5 \times 10^{-6}$.

corresponds to choosing a restitution coefficient, defined by (2.9), close to zero. The continuous problem energy will be conserved if and only if $e=1$, which is a totally elastic shock. The results we obtain are then mechanically consistent. To conclude, when the time step decreases, the loss of energy decreases too, which tends to show that the scheme creates numerical damping that is too big. Looking for energyconserving schemes for plates, as we did for beams in Pozzolini \& Salaun (2011), and also studying 


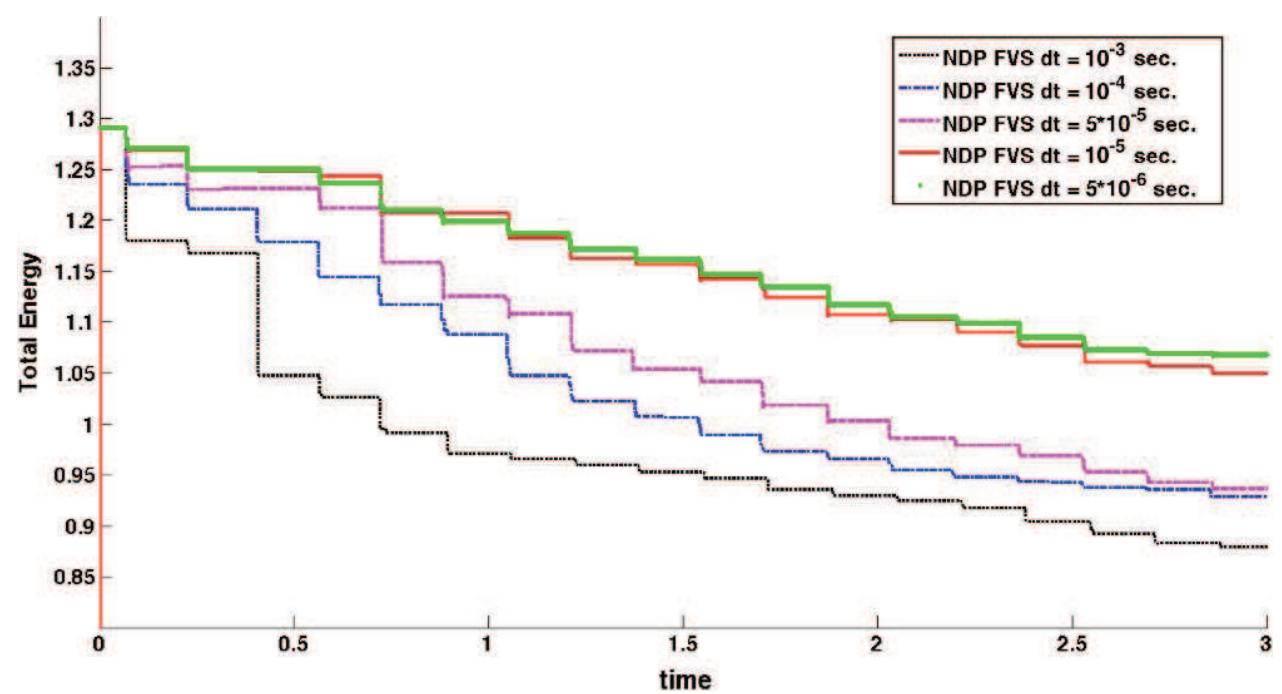

FIG. 17. Total energy variations for different values of $\Delta t-140$ FVS quadrilaterals.

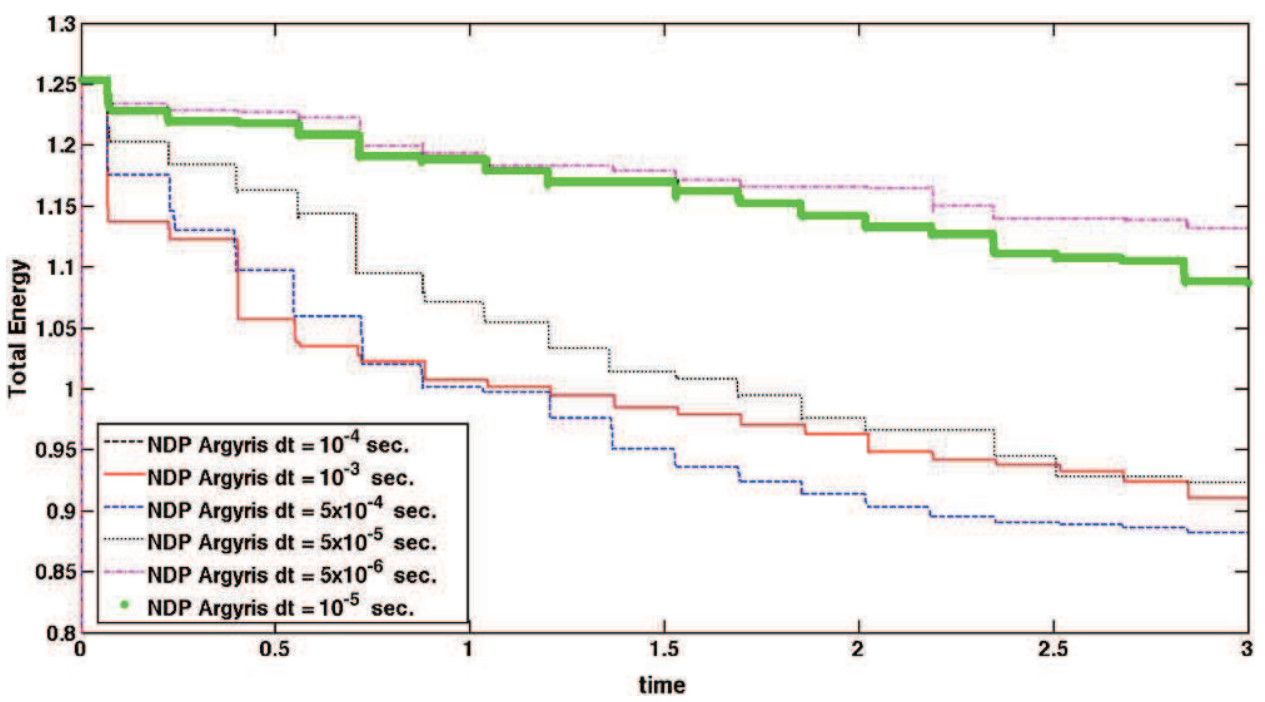

FIG. 18. Total energy variations for different values of $\Delta t-160$ Argyris triangles.

their convergence properties, is then of particular importance and will be the subject of forthcoming papers.

\section{Acknowledgements}

The work presented in this paper has been carried out with the generous support of the French Space Agency (Centre National des Etudes Spatiales de Toulouse) and of the Institut Camille Jordan (CNRS, France). 


\section{REFERENCES}

Ahn, J. \& STEWART, D. E. (2005) An Euler-Bernoulli beam with dynamic contact: Discretization, convergence and numerical results. SIAM J. Numer. Anal., 43, 1455-1480.

BOCK, I. \& JARUS̆EK, J. (2008a) On the hyperbolic contact problem for a von Karman plate. International Conference on Mathematics and Continuum Mechanics (Coimbra, Portugal), Proceedings. Coimbra, Portugal: CIM, pp. 85-91.

Bock, I. \& JARUŠEK, J. (2008b) On nonstationary von Karman variational inequalities. Tatra Mt. Math. Publ., 38, 11-28.

Brenner, S. C., Sung, L. Y. \& Zhang, Y. (2012) Finite element methods for the displacement obstacle of clamped plates. Math. Comp. (in press).

Ciarlet, P. G. (1978) The Finite Element Method for Elliptic Problems. Amsterdam, Netherland: North-Holland.

Ciarlet, P. G. (1991) Basic error estimates for elliptic problems. Handbook of Numerical Analysis, vol. II. Amsterdam, Netherland: North-Holland, pp. 17-351.

Dumont, Y. \& PAOLI, L. (2006) Vibrations of a beam between stops: convergence of a fully discretized approximation. ESAIM Math. Model. Numer. Anal., 40, 705-734.

Dumont, Y. \& PAOLI, L. (2008) Numerical simulation of a model of vibrations with joint clearance. Int. J. Comput. Appl. Technol., 33, 41-53.

Ern, A. \& Guermond, J.-L. (2004) Theory and Practice of Finite Elements. Applied Mathematical Series, vol. 159. New York: Springer.

Maury, B. (2010) Introduction à la Méthode des Éléments Finis en Élasticité. Palaiseau, France: Ecole Polytechnique.

PaOli, L. (2001) Time discretization of vibro-impact. Phil. Trans. R. Soc. Lond. A, 359, 2405-2428.

PaOli, L. \& Schatzman, M. (2007) Numerical simulation of the dynamics of an impacting bar. Comput. Methods Appl. Mech. Eng., 196, 2839-2851.

Pozzolini, C. \& SAlaun, M. (2011) Some energy conservative schemes for vibro-impacts of a beam on rigid obstacles. ESAIM Math. Model. Numer. Anal., 45, 1163-1192.

Renard, Y. \& Pommier, J. (2003-2012) An Open Source Generic C++ Library for Finite Element Methods. Available at http://home.gna.org/getfem/.

Simon, J. (1987) Compact sets in the space $L^{p}(0, T ; B)$. Ann. Mat. Pura Applic., 146, 65-96. 